\title{
XVIII. The oscillations about a position of equilibrium where a simple linear relation exists between the frequencies of the principal vibrations
}

\author{
H.J.E. Beth
}

To cite this article: H.J.E. Beth (1913) XVIII. The oscillations about a position of equilibrium where a simple linear relation exists between the frequencies of the principal vibrations, Philosophical Magazine Series 6, 26:152, 268-324, DOI: 10.1080/14786441308634973

To link to this article: http://dx.doi.org/10.1080/14786441308634973

曲 Published online: 08 Apr 2009.

6 Submit your article to this journal 준

Џلll Article views: 3

Q View related articles ¿

4 Citing articles: 9 View citing articles 5 


\section{$\left[\begin{array}{lll}{[} & 268 & ]\end{array}\right.$}

XVIII. The Oscillations about a Position of Equilibrium where a Simple Linear Relation exists hetween the Frequencies of the Principal Vilrations. By H. J. E. BEтH *.

[Plates VII. \& VIII.]

\$1. TOR a mechanism with $m$ degrees of freedom performing small oscillations about a position of stable equilibrium the equations of motion may be written in the form :

$$
\left.\begin{array}{rl}
\ddot{x}+n_{x}^{2} x+\sum_{g} \dot{x}^{2}+\sum_{g} \dot{x} \dot{y}+\sum_{g} \ddot{x}+\sum_{g} u \ddot{y}+\sum_{g} x^{2}+\sum_{g} x y+\sum_{g} x \dot{x}^{2}+\ldots \ldots & =0 \\
\ddot{y}+n_{y}{ }^{2} y+\sum_{g} \dot{x}^{2}+\ldots \ldots & =0 \\
\ldots \ldots \ldots \ldots \ldots & \ldots \ldots \ldots
\end{array}\right\}
$$

where $x, y$, etc. are the principal coordinates of the mechanism; they vanish in the position of equilibrium; $\sum_{g} i^{2}$ is written instead of $a \dot{x}^{2}+b \dot{y}^{2}+c z^{2}+\ldots$ etc.; $n_{x}, n_{y}$, etc. are the frequencies of the principal vibrations.

In his treatise $+:$ "On certain vibrations of higher order of abnormal intensity (vibrations of relation) for mechanisms with more degrees of freedom " (Verhandelingen der Koninklyke Akademie van Wetenschappen, vol. v. No. 8, 1897 ; Archives Neerlandaises, vol. i. series 2, pp. 229-260), Prof. Korteweg has shown that the coordinates $x, y \ldots$ may be expressed by the following expansions in series:
(2)
(3)

$x=\underset{00 \ldots 0}{\alpha}+\mathrm{A} h \cos \phi+\underset{010 \ldots 0}{\alpha} \cos \psi \underset{001 \ldots 0}{\alpha} \cos \chi+\ldots$

$$
+\underset{p q r \ldots}{\stackrel{(S)}{\alpha} \cos }(p \phi+q \psi+r \chi+\ldots)
$$

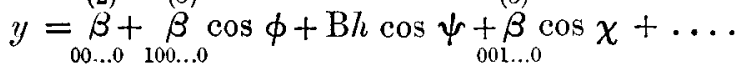

$$
\begin{aligned}
& +\underset{p q r \ldots}{\stackrel{(\mathrm{S})}{\beta} \cos (p \phi+q \psi+r \chi+\ldots)})
\end{aligned}
$$

- Communicated by the Author.

+ "Over zekere trillingen van hooger orde van abnormale intensiteit (relatietrillingen) bij wechanismen met meerdere graden van vrijheid." 
where

$$
\dot{\phi}=\left(n_{x}+\stackrel{(2)}{\sigma)} t+\lambda ; \psi=\left(n_{y}+\tau\right) t+\mu ; \ldots\right.
$$

(r) (2) (2)

$\alpha, \quad \sigma, \tau, \ldots$ are functions of $h$ of this form :

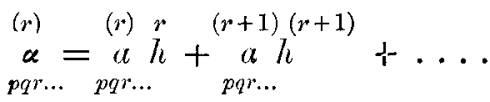

$$
\begin{aligned}
& \stackrel{(2)}{\sigma}=\stackrel{(2)}{s} h^{2}+\ldots . \\
& \text { (2) (2) } \\
& \tau=t l l^{2}+\ldots .
\end{aligned}
$$

$\mathrm{A} h, \mathrm{~B} h, \ldots . \lambda, \mu, \nu \ldots$. are constants of integration; $\mathrm{A}, \mathrm{B}, \mathrm{C}$.... are of moderate greatness; $h$ is a quantity which is small in respect to a certain greatness $l$, depending $e . q$. on the dimensions of the mechanism.

$\mathrm{S}$ is the sum of the absolute values of $p, q, r \ldots$

In order to prove this proposition, the expansions (2) were substituted in the differential equations; the products and powers of the cosines and sines were written in the form of sums of cosines and constant terms; then the sum of the terms with the same expression $\cos (p \phi+q \psi+\ldots)$ having as coefficient a function of $h$, the coefficient of each power of $h$ may be equalized to zero.

By substituting as before we find for the coefficient of $\cos (p \phi+q \psi+\ldots)$ the following expression :

$$
\left.\left[-\left\{\left(p n_{x}+q n_{y}+\ldots\right)+(p) \stackrel{(2)}{(2)} \sigma+q \tau+\ldots\right)\right\}^{2}+n_{x}^{2}+\Sigma \mathrm{A}^{2} l^{2}+\ldots\right]_{p q r . .}^{\stackrel{(\mathrm{S})}{\alpha}}
$$

+ other terms containing products of $\alpha$ 's, $\beta$ 's, etc.

(the terms $\sum \mathrm{A}^{2} h^{2}$ originate from terms as $e \cdot g$.

$$
\left.\cos ^{2} \phi \cos (p \phi+q \psi+\ldots)\right) \text {. }
$$

Supposing the coefficients in the expressions $\sigma, \tau \ldots$. to have been calculated already, we may write the coefficient of $\alpha \cos (p \phi+q \psi+\ldots)$ in the form :

$$
\xi \stackrel{p q \ldots}{\equiv}\left\{(p-1) n_{x}+q n_{y}+\ldots\right\}\left\{(p+1) n_{x}+q n_{y}+\ldots\right\}+\mathrm{D} h^{2}+\ldots
$$

The remaining terms which appear and which we $\operatorname{suppos} \theta$ to have been arranged according to powers of $h$, do not (S) contain $\alpha$ in their coefficients, but products of $\alpha$ 's, $\beta$ 's, ...., which may have been calculated from preceding equations 
obtained by equalizing to zero the coefficients of smaller powers of $h$ in terms containing factors $\cos \left(p^{\prime} \phi+q^{\prime} \psi+\ldots\right)$. We shall call the sum of the coefficients of these remaining terms P. Then we have: pqr...

Therefore :

$$
\underset{p q r \ldots}{\xi} \underset{p q r \ldots}{\stackrel{(\mathrm{S})}{\alpha}}=\underset{p q r \ldots}{\mathrm{P}}
$$

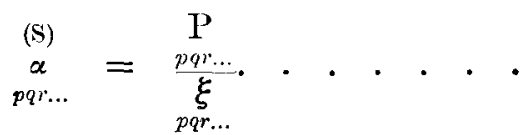

From (3) it follows that it is possible that $\xi$ takes an $p q$.

abnormally small value ; in this case according to (4) $\alpha$ takes an abnormally great value. This will happen when one of the expressions

$$
\begin{aligned}
& (p-1) n_{x}+q n_{y}+\ldots \ldots \\
& (p+1) n_{x}+q n_{y}+\ldots \ldots
\end{aligned}
$$

becomes small, or in other words when there exists a relation between $n_{x}, n_{y}$, etc. of the form :

$$
p_{1} n_{x}+q_{1} n_{y}+\ldots \ldots=\rho, \text {. . . . . • }
$$

where $p_{1}, q_{1}$, etc. are positive or negative integers, and where $\rho$ is with respect to $n_{x}, n_{y}$, etc. a small quantity, which we call residue of relation.

When $k$ is an integer, then

$$
k p_{1} n_{x}+k q_{1} n_{y}+\ldots
$$

is also small (we suppose $k$ not to be very great).

If now in (3)

or if

$$
p-1=k p_{1}, \quad q=k g_{1}, \ldots
$$

$$
p+1=k p_{1}, \quad q=k q_{1}, \ldots
$$

then $\underset{p q r . .}{\xi}$ becomes very small.

We see that there are in that case among the terms of higher order terms with an abnormally great coefficient in consequence of the relation (5) ; they represent vibrations of abnormally great intensity, which are called vibrations of relation. By vibrations of relation of the first kind we miderstand such as correspond to

$$
n=k p_{1}-1, \quad q=k q_{1}, \ldots
$$


The vibrations of relation of the second kind correspond to

$$
p=k p_{1}+1, \quad q=k_{q_{1}}, \ldots
$$

The vibrations of relation, which correspond to different values of $k$, are called of different degree.

The frequencies of the vibrations of relation of the first and second kind and of the $k$ th degree are resp. $n_{x}+k \rho+\mathrm{E} h^{2}$ and $n_{x}-k \rho-\mathrm{E} h^{2}$, where $\mathbf{E}$ depends on the values of $\mathrm{A}, \mathrm{B}$, etc.

What now is found for the coordinate $x$ is also exact for $y$ and for all the coordinates which enter in the relation (5). So we may say:

When there exists a linear relation of the form (5) then there are a number of vibrations of higher order, which assume an abnormally great intensity; their frequencies lie in the vicinity of and on both sides of the principal frequencies which appear in the relation.

\$2. Prof. Korteweg now investigates to which order of greatness the vibrations of relation will rise in general. This depends on the intensity of the motion of the mechanism, $i . e$, on $h$. $\xi$ takes the value $\rho \mathrm{Q}+\mathrm{D} h^{2}$. If now the intensity of the motion is so great that $h^{2}$ becumes of the same or of smaller order than $\rho$, then we may in (4) divide the numerator and the denominator by $h^{2} ;$ so $\alpha$ has risen two orders of greatness. This is exact for all vibrations of relation of both kinds and every degree. So we have now but to examine the function $P$.

The function $\underset{p q . .}{\mathrm{P}}$ may rise in greatness in consequence of those terms appearing in $\mathrm{P}$, which have themselves risen in greatness, $i . e$. have become of a smaller order than in the general case where no relation exists. This will not occur for the vibration of relation of the first kind and first degree. It will occur, however, for the vibration of the second kind and first degree, in consequence of terms as e. $g$.

$$
\cos ^{2} \phi \cos ((p-1) \phi+q \psi+\ldots) \text {. }
$$

This term having risen two orders, $P$ will also rise two orders. So the term of the second kind and first degree will rise four orders, while that of the first kind and first degree will rise only two orders. The order of the former being in general $S_{1}+1$, that of the second $S_{1}-1$, both terms will rise to the same order, namely $S_{1}-3$.

Now we pass on to the vibrations of relation of the second degree. In the $\mathrm{P}$ function for the term of the first kind 
appear among other terms those of the first degree and second kind, which have risen 4 orders. So this term rises $2+4=6$ orders. The $P$ function for the term of second degree and second kind rises 6 orders also; so the term rises $2+6=8$ orders. Both terms of the second degree rise to the same order.

In this way we may go on. It will be clear that hoth terms of the same degree rise to the same order. Each following term rises 4 orders more than the former. The order of the term of the first kind and $k$ th degree being in general $k \mathrm{~S}_{1}-1$, the order of both terms of the $k$ th degree will become

$$
k \mathrm{~S}_{1}-1-(k-1) 4-2=k\left(\mathrm{~S}_{1}-4\right)+1 .
$$

$\$ 3$. From this result it follows immediately that there exists a considerable difference between the two cases $\mathrm{S}_{1}<4$ and $S_{1}>4$. In the latter case the vibrations of relation remain feeble in respect to the principal vibrations; the intensity is smalier for the vibrations of a higher degree.

In the case $S_{1}=4$, however, all the vibrations of relation reach the order of greatness of the principal vibrations. In the cases $S_{1}=3$ and $S_{1}=2$, it should seem as if the vibrations of relation would have an intensity which is great in respect to the intensity of the principal vibrations. This conclusion is not exact, as Prof. Korteweg shows by a special investigation into these cases. However, it will be clear that the development in series (2) have lost their validity completely as soon as the intensity of the motion has become so great that the vibrations of relation reach the intensity of the principal vibrations.

Therefore in the cases $S_{1}<4$, as soon as the motion of the mechanism has a certain intensity the developments in series can no longer represent the motion. In what follows we shall investigate what becomes of the motion in the case mentioned.

In Prof. Korteweg's paper it is shown that the developments lose their validity in the case $\mathrm{S}_{1}=3$ as soon as $\frac{h}{l}$ is of the same order of greatness as $\frac{\rho}{n_{x}}$; in the cases $\mathrm{S}_{1}=4$ and $\mathrm{S}_{1}=2$ as soon as $\left(\frac{l}{l}\right)^{2}$ is of the same order of greatness as $\frac{\rho}{n_{x}}$. Therefore we shall suppose $\frac{\rho}{n_{x}}$ to be of order $\frac{l}{l}$ in the case $S_{1}=3$, and of order $\left(\frac{h}{l}\right)^{2}$ in the cases $S_{1}=4$ and 
$\mathrm{S}_{1}=2$. We shall examine how by the decrease of the amplitudes, or, what is the same, by the increase of $\rho$, the transition to the general case takes place.

The difference between the cases $S_{1}>4$ and $S_{1}<4$ is one of the results Prof. Korteweg arrives at. In the foregoing lines I have tried to repeat the main points of his reasoning; however, I have omitted what seemed to me to be of less importance for what will follow now. So Prof. Korteweg finds that there exists a third kind of vibrations of relation, whose frequencies lie generally in the neighbourhood of all principal frequencies, which do not appear in the relation; they behave as the vibrations of the second kind.

The investigation of the motion of a vibrating mechanism having two degrees of freedom in the cases where Prof. Korteweg's developments cease to represent this motion was the subject of my dissertation (Amsterdam, 1910). However, Prof. Korteweg had found already the nature of the motion for the case of the strict relation $n_{y}=2 n_{x}$ and the relation between $\zeta$ and $\phi$ for this case, which we shall deduce p. 286 , as well as the special cases which for this relation may occur. This solution has been my key to the examination of the other cases, though I have during the course of my investigations directed them along other lines than were traced out in Prof. Korteweg's solution. The dissertation mentioned before was published in an abridged form (Proceedings, Amsterdam, pp. 618-635, and pp. 735-750, 1910; Arclives Néerlandaises, series 2, vol. xv. pp. 246-283, 1910). In another paper (Proceedings, Amsterdam, pp. 742-761, 1911; Archives Néerlandaises, series 3 A, vol. i. pp. 185-208, 1912) I have extended the investigation to a mechanism having an arbitrary number of degrees of freedom. Some months ago in a prize-essay (not yet published) written in answer to a problem set by the Mathematical Society of Amsterdam, I have examined the envelope of systems of Lissajous' curves which occur in the question we have in view ; in my dissertation the envelope had been found only for the case $n_{y}=2 n_{x}+\rho$. The subject having come so far to a conclusion it may appear desirable to publish a new summary of the problem.

$\S 4$. In case a relation of the form

$$
p_{1} n_{x}+g_{1} n_{y}+\ldots \ldots=\rho
$$

exists, some terms in the equations of motion, after substitution of the expressions which represent in the general case the coordinates at first approximation, give rise to terms of 
nearly the same period as the principal vibration, to which the equation in which the term indicated appears, relates more especially. Such terms, as is known, may be of importance, even if they are of a high order; it may occur that they are of influence even on the first approximation. In order to determine the motion at first approximation it will be necessary to include such terms in the abridged equations of motion.

Now we must notice that terms of this kind appear in the equations of motion even when no relation exists. The term $x y^{2}$ for instance will after substitution as above give rise to a term of the same period as $x$. That these terms are of influence on the first approximation we see from the developments $(2, \mathrm{p} .268)$. The modification of the frequencies, indicated by $\sigma, \tau, \ldots$ is the effect of the terms we refer to. These terms are of order $h^{3}$ at least.

We shall call disturbing terms such terms of higher order which are of importance for the first approximation. It follows from what is said above that we have to distinguish :

1st, the terms which are always disturbing, even when no relation exists; we shall call them disturbing terms of the first kind.

2nd, the terms which owe their disturbing property to the existing relation; we shall call them disturbing terms of the second kind.

In a single case $\left(S_{1}=2\right)$ there appear terms which are disturbing in both senses.

In order to obtain the first approximation we shall admit in the differential equations of the terms of higher order only the disturbing ones, and of these terms only those of the lowest order. We shall prove that in each of the cases we have to consider, the equations of motion may be brought into this form :

$$
\begin{aligned}
& \ddot{x}+n_{x}^{2} x-\frac{\partial \mathrm{R}}{\partial x}=0, \\
& \ddot{y}+n_{y y}^{2} y-\frac{\partial \mathrm{R}}{\partial y}=0,
\end{aligned}
$$

where $\mathrm{R}$ is a function of $x, y, \ldots$ T'o prove this, and for the deduction of the function $R$, we shall successively discuss the cases $S_{1}=3, S_{1}=4$, and $S_{1}=2$. 


$$
S_{1}=3 \text {. }
$$

$\S 5$. There are two relations to be considered, namely :

$$
\begin{array}{ll}
1^{\circ}, & 2 n_{x}-n_{y}=\rho, \\
2^{\circ}, & n_{x}+n_{y}-n_{z}=\rho .
\end{array}
$$

When one of these relations exists there are in the equations of motion disturbing terms of the second kind of urder $h^{2}$, e. $g$. the term with $x y$ in the first equation in case of the first relation. So the disturbing terms of the first kind can be left out, because they are of order $h^{3}$ at least, and because we admit the disturbing terms of the lowest order onlr.

It is clear that the disturbing terms of the second lind of order $h^{2}$ appear only in the equations resp. for $x$ and $y$, and for $a, y$, and $z$. They contain no other but these coordinates. So we may say:

In the case $\mathrm{S}_{1}=3$ the coordinates which appear in the relation behave at first approximation as if they were the only coordinates; the remaining coordinates behave at first approximation as if no relation existed.

It will therefore be sufficient to consider a mecbanism which has 2 , resp. 3 degrees of freedom.

$\S 6.2 n_{x}-n_{y}=\rho$. As we have to include in the differential equations no terms of a higher order than $h^{2}$, we have in the expressions for the kinetic energy $\mathrm{T}$ and for the potential energy $U$ to take no terms of a higher order than $h^{3}$. So we may write :

$$
\mathrm{T}=\frac{1}{2} \dot{x}^{2}+\frac{1}{2} \dot{y}^{2}+\mathrm{T}_{3} ; \quad \mathrm{U}=\frac{1}{2} n_{x}^{2} x^{2}+\frac{1}{2} n_{y}{ }^{2} y^{2}+\mathrm{U}_{3},
$$

where $\mathrm{U}_{3}$ is a homogeneous function of order three in $x, y$, and

$$
\mathrm{T}_{3}=\frac{1}{2}\left(a x \dot{x}^{2}+b y \dot{x}^{2}+2 c x \dot{x} \dot{y}+2 d y \dot{x} \dot{y}+e x \dot{y}^{2}+f y \dot{y}^{2}\right) \text {. }
$$

The equations of Lagrange become :

$$
\left\{\begin{array}{l}
\ddot{x}+n_{x}{ }^{2} x=-\frac{1}{2} a \dot{x}^{2}-a \ddot{x} \ddot{x}-b y \ddot{x}-b \dot{x} \dot{y}-c x \ddot{y}-d y \ddot{y}+\left(\frac{1}{2} e-d\right) \dot{y}^{2}-\frac{\partial \mathrm{U}_{3}}{\partial x} \\
\ddot{y}+n_{y}{ }^{2} y=\left(\frac{1}{2} b-c\right) \dot{x}^{2}-c \ddot{x}-d y \ddot{x}-e \dot{x} \dot{y}-e x \ddot{y}-f \ddot{y} \ddot{y}-\frac{1}{2} f \ddot{y}^{2}-\frac{\partial \mathrm{U}_{3}}{\partial y} .
\end{array}\right.
$$

The disturbing terms are:

in the first equation those with $y \ddot{x}, \dot{x} \dot{y}, x \ddot{y}, x y$ :

" second ", " " $\quad \dot{x}^{2}, x \ddot{x}, x^{2}$.

Now we shall try to satisfy the equations in first approximation by

$$
x=\mathrm{A} h \cos \left(n_{x} t+\lambda\right), \quad y=\mathrm{B} h \cos \left(n_{y} t+\mu\right),
$$

where $\mathrm{A}, \mathrm{B}, \lambda$, and $\mu$ are functions of $t$, however in such a 
manner, that $\dot{A}, \dot{B}, \dot{\lambda}$, and $\dot{\mu}$ are of order $h$ or smaller. Then we may replace in the second member of the equations:

$$
\begin{array}{ll}
\dot{x}^{2} \text { by } n_{x}{ }^{2}\left(\mathrm{~A}^{2} h^{2}-x^{2}\right), & \dot{y}^{2} \text { by } n_{y}{ }^{2}\left(\mathrm{~B}^{2} h^{2}-y^{2}\right), \\
\ddot{x},-n_{x}{ }^{2} x, & \ddot{y},,-n_{y}{ }^{2} y .
\end{array}
$$

If we take this into account for the disturbing terms and if we omit the non-disturbing terms, then the equations become :

$$
\left\{\begin{array}{l}
\ddot{x}+n_{x}{ }^{2} x=\left(b n_{x}{ }^{2}+c n_{y}{ }^{2}+2 p\right) x y-b \dot{x} \dot{y}, \\
\ddot{y}+n_{y}{ }^{2} y=\left(2 c n_{x}{ }^{2}-\frac{1}{2} l n_{x}{ }^{2}+p\right) x^{2} .
\end{array}\right.
$$

(The terms 2pxy in the first equation and $p x^{2}$ in the second originate from a term $-p x^{2} y$, appearing in $\mathrm{U}_{3}$.)

'To get rid of the term with $x \dot{y}$ we notice that $\dot{x} \dot{y}$ by substitution of the expressions for $x$ and $y$ gives rise to the same disturbing term as $n_{x} n_{y} x y$. So we may replace $-b \dot{x} y$ hy $-l n_{x} n_{y} x y$.

We replace in the terms of higher order $n_{y}$ by $2 n_{x^{*}}$. Then the equations become :

$$
\left\{\begin{array}{l}
\ddot{x}+n_{x}^{2} x=\left(4 c n_{x}^{2}-b m_{x}^{2}+2 p\right) x y, \\
\ddot{y}+n_{y}^{2} y=\left(2 c n_{x}^{2}-\frac{1}{2} 6 n_{x}^{2}+p\right) x^{2} .
\end{array}\right.
$$

We may write the equations in the form:

$$
\left\{\begin{array}{l}
\ddot{x}+n_{x}^{2} x-\frac{\partial \mathrm{R}}{\partial x}=0, \\
\ddot{y}+n^{2} y-\frac{\partial \mathrm{R}}{\partial y}=0,
\end{array}\right.
$$

where, if we write $-d_{2}$ for $2 n_{x}^{2}-\frac{1}{2} b m_{x}^{2}+p$ :

$$
\mathrm{R}=-d_{2} x^{2} \%
$$

$\S 7 . n_{x}+n_{y}-n_{z}=\rho . \quad$ In this case

$$
\mathrm{U}=\frac{1}{2} n_{x}^{2} x^{2}+\frac{1}{2} n_{y}^{2} y^{2}+\frac{1}{2} n_{z}^{2} z^{2}+\mathrm{U}_{3},
$$

where $\mathrm{U}_{3}$ is a homogeneous function of order three in $x, y$, and $z$.

$$
\mathrm{T}=\frac{1}{2} \dot{x}^{2}+\frac{1}{2} \dot{y}^{2}+\frac{1}{2} \dot{z}^{2}+\frac{1}{2}\left(\mathrm{P}_{x x^{2}} \dot{x}^{2}+2 \mathrm{P}_{x y} \dot{i x} \dot{y}+\ldots\right),
$$

where e. $g$.

$$
\mathrm{P}_{x y}=a_{x y} x+b_{x y} y+c_{x y}=
$$

The equations of Lagrange can be written down again. When in the terms of the 2nd order $\ddot{x}, \ddot{y}$, and $\ddot{z}$ are replaced resp. by $-n_{x}{ }^{2} x,-n_{y}{ }^{2} y$, and $-n_{z}{ }^{2} z$, then we have $e . g$. in the 
equation for $x$ to consider as disturbing the terms with $y z$ and $\dot{y} \dot{z}$. We may replace $\dot{y} \dot{z}$ by $n_{y} n_{z} y z$, and in the terms of the 2 nd order we may put $n_{x}+n_{y}-n_{z}=0$. In this way we arrive at the following form for the first equation :

$$
\ddot{x}+n_{x}^{2} x=\left(a_{y z} n_{y} n_{z}+b_{x z} n_{x} n_{z}-c_{x y} n_{x} n_{y}-p\right) y z
$$

( $p$ being the coefficient of the term $x y z$ in $\mathrm{U}_{3}$ ).

In the same way we can also simplify the two remaining equations; we must bear in mind that in the second equation $x \dot{z}$ must be replaced by $n_{x} n_{z} x z$, in the third equation however $\dot{x} \dot{y}$ by $-n_{x} n_{y} x y$. form :

The result is that the equations may be written in this

$$
\left\{\begin{array}{l}
\ddot{x}+n_{x}^{2} x-\frac{\partial \mathrm{R}}{\partial z}=0, \\
\ddot{y}+n_{y}^{2} y-\frac{\partial \mathrm{R}}{\partial y}=0, \\
\ddot{z}+n_{z}^{2} z-\frac{\partial \mathrm{R}}{\partial z}=0 ;
\end{array}\right.
$$

where, if $p^{\prime}$ is written instead of $p-\left(a_{y z} n_{y} n_{z}+b_{x z} n_{x} n_{z}-c_{x y} n_{x} n_{y y}\right)$

$$
\begin{gathered}
\mathrm{R}=-p^{\prime} x y z . \\
\mathrm{S}_{1}=4 .
\end{gathered}
$$

$\S 8$. There are five relations to be considered, namely :

$$
\begin{aligned}
3 n_{x}-n_{y} & =\rho, \\
\pm n_{x}+2 n_{y}-n_{z} & =\rho, \\
n_{x}+n_{y} \pm n_{z}-n_{u} & =\rho .
\end{aligned}
$$

In the equations of motion no term of the second urder is disturbing. So the terms of the second order may in the equations be left out; in $T$ and $U$ the terms of the third order may be left out. Among the terms of the third order in the equations are disturbing terms as well of the first kind as of the second kind. Terms of higher order than $h^{3}$ we may omit. The disturbing terms of the second kind are different in the different cases mentioned above; those of the first kind are always the same. We shall first discuss these terms.

It is clear that disturbing terms of the first kind may appear in all equations and that they will contain in general all coordinates. In the equation for $x$ disturbing terms of the first kind of order $h^{3}$ are those with : $x^{3}, x^{2} \cdot, x \dot{x}^{2}, x y^{2}$, $x z^{2}, \ldots \ldots, \ddot{x} y^{2}, \ddot{x} z^{2}, \ldots \ldots, x \dot{y}^{2}, x \dot{z}^{2}, \ldots \ldots, x y \ddot{y}, x z \ddot{z}, \ldots \ldots$. 
If we take again as a first approximation :

$$
x=\mathrm{A} h \cos \left(n_{x} t+\lambda\right), \quad y=\mathrm{B} h \cos \left(n_{y} t+\mu\right), \ldots \ldots,
$$

in which $\mathrm{A}, \mathrm{B}, \ldots \lambda, \mu, \ldots$ are functions of $t$ in such a manner that $\dot{\mathrm{A}}, \dot{\mathrm{B}}, \ldots \dot{\lambda}, \dot{\mu}, \ldots$ are of order $h^{2}$ or smaller, then in the terms of higher order we may substitute $-n_{x}^{2} x$ for $\ddot{x}$, $-n_{y}^{2} y$ for $\ddot{y}, \ldots, \mathrm{A}^{2} l^{2}-n_{x}{ }^{2} x^{2}$ for $\dot{x}^{2}, \mathrm{~B}^{2} h^{2}-n_{y}{ }^{2} y^{2}$ for $\ddot{y}^{2}, \ldots \ldots$ We then retain as disturbing terms of the first kind in the first equation only those with

$$
\mathrm{A}^{2} h^{2} x, \mathrm{~B}^{2} h^{2} x, \ldots \ldots, x^{3}, x y^{2}, \ldots \ldots
$$

Now we replace $x y^{2}$ by $\frac{\mathrm{B}^{2} h_{L}^{2}}{2} x$, etc., which is permitted because they give rise to the same disturbing terms.

If we reduce in the same way the disturbing terms of the first kind in the remaining equations, we shall find that these terms are in the different equations the derivatives resp. according to $x, y, \ldots \ldots$ of :

$\mathrm{K}_{x} x^{4}+\mathrm{K}_{y} y^{4}+\ldots \ldots+\mathrm{M}_{x x} \mathrm{~A}^{2} h^{2} x^{2}+\mathrm{M}_{y x} \mathrm{~B}^{2} h^{2} x^{2}+\mathrm{M}_{x y} \mathrm{~A}^{2} h^{2} y^{2}+\ldots \ldots$, where the K's and M's are coefficients.

About the disturbing terms of the second kind we may make the following general observation. They appear only in the equations for those coordinates which enter in the relation, and they contain no other coordinates. So we may, in order to determine these terms, restrict ourselves respectively to mechanisms with 2,3 , or 4 degrees of freedom. We now shall discuss these terms for the different relations separately.

§ $9.3 n_{x}-n_{y}=\rho .-$ In this case :

where

$$
\mathrm{T}=\frac{1}{2} \dot{x}^{2}+\frac{1}{2} \dot{y}^{2}+\frac{1}{2} \mathrm{P}_{x x} \dot{x}^{2}+\mathrm{P}_{x y} x \dot{y}+\frac{1}{2} \mathrm{P}_{y y} \dot{y}^{2}
$$

Further

$$
\begin{aligned}
& \mathrm{P}_{x x}=\frac{1}{2} a_{x x} x^{2}+b_{x x} x y+\frac{1}{2} c_{x x} y^{2}, \\
& \mathrm{P}_{x y}=\frac{1}{2} a_{x y} x^{2}+b_{x y} x y+\frac{1}{2} c_{x y} y^{2}, \\
& \mathrm{P}_{y y}=\frac{1}{2} a_{y y} x^{2}+b_{y y} x y+\frac{1}{2} c_{y y} y^{2} .
\end{aligned}
$$

$$
\mathrm{U}=\frac{1}{2} n_{x}^{2} x^{2}+\frac{1}{2} n_{y}^{2} y^{2}+\mathrm{U}_{4},
$$

where $U_{4}$ represents a homogeneous function of degree 4 in $x$ and $y$.

When in the same way as is done for the former cases the disturbing terms in the different equations are reduced, 
we find that they are the derivatives, resp. according to $x, y$, of

$$
\left\{\left(-\frac{1}{2} b_{x x}+\frac{3}{2} a_{x y}\right) n_{x}{ }^{2}-l\right\} x^{3} y .
$$

( $l$ is the coefficient of the term $x^{3} y$ in $\mathrm{U}_{1}$ )

So the equations of motion may be written in this form :

$$
\left\{\begin{array}{l}
\ddot{x}+n_{x}^{2} x-\frac{\partial \mathrm{R}}{\partial x}=0, \\
\ddot{y}+n_{y}{ }^{2} y-\frac{\partial \mathrm{R}}{\partial y}=0 ;
\end{array}\right.
$$

where, if $-f$ is written instead of $\left(-\frac{1}{2} l_{x x}+\frac{3}{2} a_{x y}\right) n_{x}{ }^{2}-l$ :

$$
\begin{aligned}
& \mathrm{R}=\mathrm{K}_{x} x^{4}+\mathrm{K}_{y} y^{4}+\ldots \ldots+\mathrm{M}_{x x} \mathrm{~A}^{2} h^{2} x^{2}+\mathrm{M}_{y x} \mathrm{~B}^{2} h^{2} x^{2}+\ldots \ldots-f x^{3} y . \\
& \quad \S 10 . \quad \pm n_{x}+2 n_{y}-n_{z}=\rho .-\mathrm{In} \text { this case }: \\
& \mathrm{T}=\frac{1}{2} \dot{x}^{2}+\frac{1}{2} \dot{y}^{2}+\frac{1}{2} \dot{z}^{2}+\frac{1}{2} \mathrm{P}_{x x} \dot{x}^{2}+\mathrm{P}_{x y} \dot{x} \dot{y}+\ldots \ldots,
\end{aligned}
$$

where

$$
\begin{aligned}
& \mathrm{P}_{x x}=\frac{1}{2} a_{x x} x^{2}+\frac{1}{2} b_{x x} y^{2}+\frac{1}{2} c_{x x} z^{2}+e_{x x} x y+f_{x x} x z+h_{x x} y z \\
& \mathrm{P}_{x y}=\frac{1}{2} a_{x y} x^{2}+\frac{1}{2} b_{x y} y^{2}+\frac{1}{2} c_{x y} z^{2}+e_{x y} x y+f_{x y} x z+h_{x y} y z .
\end{aligned}
$$

Further

$$
\mathrm{U}=\frac{1}{2} n_{x}^{2} x^{2}+\frac{1}{2} n_{y}^{2} y^{2}+\frac{1}{2} n_{z}^{2} z^{2}+\mathrm{U}_{4},
$$

where $\mathrm{U}_{4}$ represents a homogeneous function of degree 4 in $x, y$, and $z$. The result is that the disturbing terms of the second kind in the different equations after reduction are the derivatives, resp. according to $x, y$, and $z$ of :

$$
\left(\mp n_{x} n_{y} h_{x y} \pm n_{x} n_{z} b_{x z}-\frac{1}{2} n_{y}^{2} f_{y y}+n_{y} n_{z} e_{y z}-p\right) x y^{2} z \text {. }
$$

( $p$ is the coefficient of the term $x y^{2} z$ in $U_{4}$.)

Therefore the equations of motion may be written as follows :

$$
\begin{aligned}
& \ddot{x}+n_{x}^{2} x-\frac{\partial \mathrm{R}}{\partial x}=0, \\
& \ddot{y}+n_{y}^{2} y-\frac{\partial \mathrm{R}}{\partial y}=0, \\
& \ddot{z}+n_{z}^{2} z-\frac{\partial \mathrm{R}}{\partial z}=0
\end{aligned}
$$

where, if $-f$ is writien instead of $\mp n_{x} n_{y} h_{x y} \pm n_{x} n_{z} b_{x z}$ $-\frac{1}{2} n_{y}^{2} f_{y y}+n_{y} n_{z} e_{y z}-p$ :

$$
\mathrm{R}=\mathrm{K}_{x} x^{4}+\mathrm{K}_{y} y^{4}+\ldots \ldots+\mathrm{M}_{x x} \mathrm{~A}^{2} h^{2} x^{2}+\mathrm{M}_{y x} \mathrm{~B}^{2} h^{2} x^{2}+\ldots \ldots-f x y^{2} z .
$$


\$11. $\underline{n}_{x}+n_{j y} \pm n_{z}-n_{u}=\rho .-$ In this case :

$$
\mathrm{T}=\frac{1}{2} u^{2}+\frac{1}{2} \dot{y}^{2}+\frac{1}{2} \dot{z}^{2}+\frac{1}{2} \dot{u}^{2}+\frac{1}{2} \mathrm{P}_{x x} \dot{x}^{2}+\mathrm{P}_{x y} \dot{i} \dot{y}+\ldots \ldots,
$$

where

$$
\begin{aligned}
\mathrm{P}_{x x}=\frac{1}{2} a_{x x} x^{2}+\frac{1}{2} b_{x x} y^{2}+\frac{1}{2} c_{x x z^{2}} & +\frac{1}{2} d_{x x} u u^{2}+e_{x x} x y+f_{x x} x z \\
& +g_{x x} x u+h_{x x} y z+k_{x x} y u+l_{x x} z u . \\
\mathrm{P}_{x y y}=\frac{1}{2} a_{x y} x^{2}+\frac{1}{2} b_{x y} y^{2}+\frac{1}{2} c_{x y} z^{2} & +\frac{1}{2} d_{x y} u^{2}+e_{x y} x y+f_{x y} x z \\
& +g_{x y y} x u+h_{x y} y z+k_{x y} y u+l_{x y y} \tilde{z} u
\end{aligned}
$$

Further

$$
\mathrm{U}=\frac{1}{2} n_{x}{ }^{2} x^{2}+\frac{1}{2} n_{y}{ }^{2} y^{2}+\frac{1}{2} n_{z}{ }^{2} z^{2}+\frac{1}{2} n_{u b}{ }^{2} u^{2}+\mathrm{U}_{4},
$$

where $\mathrm{U}_{4}$ represents a homogeneous function of order four in $x, y, z$ and $u$.

The result is that the disturbing terms of the second kind in the different equations are the derivatives, resp. according to $x, y, z$ and $u$ of :

$$
\left(-n_{x} n_{y} l_{x y} \bar{\top} n_{x} n_{z} k_{x z}+n_{x} n_{u} h_{x u} \mp n_{y} n_{z} g_{y z}+n_{y} n_{u} f_{y u} \pm n_{z} n_{u} e_{z u}-p\right) x y z u
$$

( $p$ being the coefficient of a term $x y z u$ in $\mathrm{U}_{4}$ ).

Therefore the equations of motion may be written as follows :

$$
\begin{aligned}
& \ddot{x}+n_{x}{ }^{2} x-\frac{\partial \mathrm{R}}{\partial x}=0, \\
& \ddot{y}+n_{y}{ }^{2} y-\frac{\partial \mathrm{R}}{\partial y}=0, \\
& \ddot{z}+n_{z}{ }^{2} z-\frac{\partial \mathrm{R}}{\partial z}=0, \\
& \ddot{u}+n_{u}{ }^{2} u-\frac{\partial \mathrm{R}}{\partial u}=0 ;
\end{aligned}
$$

where, if $-f$ is written instead of $-n_{x} n_{y} l_{x y} \mp n_{x} n_{z} k_{x z}$ $+n_{x} n_{u} h_{x u} \mp n_{y} n_{z} g_{y z}+n_{y} n_{u} f_{y u}^{\prime} \pm n_{z} n_{u} e_{z u}-p:$

$\mathrm{R}=\mathrm{K}_{x} x^{4}+\mathrm{K}_{y} y^{4}+\ldots \ldots+\mathrm{M}_{x x} \mathrm{~A}^{2} h^{2} x^{2}+\mathrm{M}_{y x} \mathrm{~B}^{2} h^{2} x^{2}+\ldots \ldots-f_{i c} y z u$.

$$
\mathrm{S}_{1}=2 \text {. }
$$

$\S 12$. The only relation which belongs to this case is :

$$
\underline{n_{x}-n_{y}=\rho} \text {. }
$$

All disturbing terms which we have to consider are of order $h^{3}$. Here the peculiarity appears that all disturbing terms of the second kind must be regarded at the same time as disturbing terms of the first kind. So a term $x y^{\prime \prime}$ gives 
rise to a term with $\cos \left(n_{x} t+\lambda\right)$ and a term with

$$
\cos \left\{\left(2 n_{y}-n_{x}\right) t+2 \mu-\lambda\right\},
$$

which both are disturbing in the equation for $x$.

The disturbing terms of the first kind are the same as for $\mathrm{S}_{1}=4$.

The disturbing terms of the second kind contain no other coordinates but $x$ and $y$ and they appear only in the equations for $x$ and $y$. Therefore we may for the discussion of these terms restrict ourselves to a mechanism of two degrees of freedom.

$\mathrm{T}$ and $\mathrm{U}$ have the same form as in the case $3 n_{x}-n_{y}=\rho$ (p. 278).

The result is that the disturbing terms of the second kind in the two equations are the derivatives resp. according to $x$ and $y$ of :

$$
\begin{array}{r}
n_{x}{ }^{2}\left\{\frac{1}{3}\left(\frac{1}{2} b_{x x}+a_{x y}\right) x^{3} y+\frac{1}{2}\left(-c_{x x}+2 b_{x y}-a_{y y}\right) x^{2} y^{2}+\frac{1}{3}\left(\frac{1}{2} b_{y y}+c_{x y}\right) x y^{3}\right\}+ \\
-e_{2} x^{3} y-e_{3} x^{2} y^{2}-e_{4} x y^{3}
\end{array}
$$

$\left(e_{2}, e_{3}\right.$, and $e_{4}$ being the coefficients of the terms with $x^{3} y, x^{2} y^{2}$, and $x y^{3}$, appearing in $\mathrm{U}_{4}$ ). form :

The equations of motion may be written in the following

$$
\left\{\begin{array}{l}
\ddot{x}+n_{x}^{2} x-\frac{\partial \mathrm{R}}{\partial x}=0, \\
\ddot{y}+n_{y}^{2} y-\frac{\partial \mathrm{R}}{\partial y}=0 ;
\end{array}\right.
$$

where, if

$$
\begin{gathered}
e_{2}{ }^{\prime}=e_{2}-\frac{1}{3} n_{x}{ }^{2}\left(\frac{1}{2} b_{x x}+a_{x y}\right), \\
e_{3}{ }^{\prime}=e_{3}+\frac{1}{2} n_{x}{ }^{2}\left(c_{x x}-2 b_{x y}+a_{y y}\right), \\
e_{4}{ }^{\prime}=e_{4}-\frac{1}{3} n_{x}{ }^{2}\left(\frac{1}{2} b_{y y}+c_{x y}\right) . \\
\mathrm{R}=\mathrm{K}_{x} x^{4}+\mathrm{K}_{y} y^{4}+\ldots \ldots \mathrm{M}_{x x} \mathrm{~A}^{2} h^{2} x^{2}+\ldots . .-e_{2}{ }^{\prime} x^{3} y-e_{3}{ }^{\prime} x^{2} y^{2}-e_{4}{ }^{\prime} x y^{5} .
\end{gathered}
$$

$\S 13$. We have now brought the equations of motion for the different cases we have to consider in this form :

$$
\left.\begin{array}{c}
\ddot{x}+n_{x}{ }^{2} x-\frac{\partial \mathrm{R}}{\partial x}=0, \\
\ddot{y}+n_{y}{ }^{2} y-\frac{\partial \mathrm{R}}{\partial y}=0 \\
\ldots \ldots . . .
\end{array}\right\} \ldots .
$$

where $\mathrm{R}$ is a function of the coordinates $x, y \ldots \ldots$; the form of $\mathrm{R}$ we have deduced for the different cases.

Plil. Mag. S. 6. Vol. 26. No. 152. Aug. 1913. 
In all the cases there is at least one of the frequencies which appears in the relation (5) (p. 270) with a coefficient -1 . Let it be $n_{v}$, then the relation may be written as follows :

$$
n_{v}=p_{1} n_{x}+q_{1} n_{y}+\ldots . .-\rho .
$$

We now introduce a frequency $n_{v}{ }^{\prime}$ in such a way that

$$
n_{v}=n_{v}{ }^{\prime}-\rho \text {. }
$$

Between the frequencies $n_{x}, n_{y} \ldots \ldots n_{v}^{\prime}$ exists the strict relation :

$$
p_{1} n_{x}+q_{1} n_{y}+\ldots \ldots-n_{v}{ }^{\prime}=0 .
$$

In the differential equations we may in the terms of higher order replace $n_{v}$ by $n_{v}{ }^{\prime}$. In the terms of order $h$ this is not permitted. The frequency $n_{v}$ appears in the equation

Now

$$
\ddot{v}+n_{v}{ }^{2} v-\frac{\partial \mathrm{R}}{\partial v}=0 \text {. }
$$

$$
n_{v}^{2}=n_{v}^{\prime 2}-2 \rho n_{v}^{\prime}+\rho^{2} .
$$

By substitution of this in the equation, we may neglect the term $\rho^{2}$. The equation takes the form

$$
\ddot{v}+n_{v}{ }^{\prime 2} v-2 \rho n_{v}{ }^{\prime} v-\frac{\partial \mathrm{R}}{\partial v}=0 .
$$

If we now admit in $\mathrm{R}$ a term $\rho n_{v}{ }^{\prime} v^{2}$, then the equations of motion hold the simple form, which is written down at the beginning of this section. However $n_{v}$ is every where replaced by $n_{v}{ }^{\prime}$; between the frequencies exists a strict relation.

$\$ 14$. In order to integrate this system of equations, we make use of the method of the variation of the canonical constants. This means, as is known, that the equations, arising when the terms $\frac{\partial \mathrm{R}}{\partial x}, \frac{\partial \mathrm{R}}{\partial y}$, etc, are omitted, first are solved, in which solution $2 m$ arbitrary constants appear (as the mechanism has $m$ degrees of treedom). We then investigate what functions of the time must be the quantities just now regarded as constante, so that the expressions for the coordinates, taken in this way, represent the solution of the complete equations containing $\frac{\partial \mathrm{R}}{\partial x}, \frac{\partial \mathrm{R}}{\partial y}, \ldots \ldots$ The equations in which $\frac{\partial \mathrm{R}}{\partial x}, \frac{\partial \mathrm{R}}{\partial y}, \ldots .$. are lacking, are solved according to the method of Hamilton-Jacobi, in order that the constants we obtain may form a canonical system.

If $\alpha_{x}, \alpha_{y}, \ldots \ldots, \beta_{x}, \beta_{y} \ldots \ldots$ are the canonical constants, 
then by substitution of the expressions found for $x, y, \ldots \ldots$ in $\mathrm{R}$ this $\mathrm{R}$ will become a function of the $\alpha$ 's, the $\beta$ 's, and $t$. The variability of the $\alpha$ 's and $\beta$ 's with the time is then given by

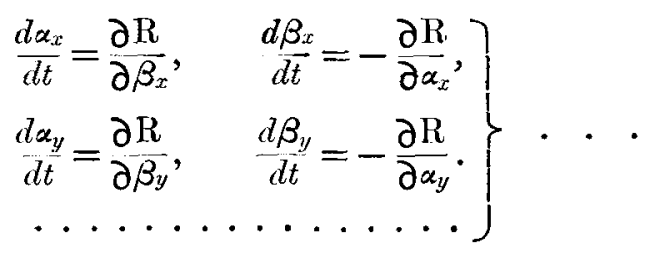

$\S 15$. If now we solve the abridged equations

$$
\left\{\begin{array}{l}
\ddot{x}+n_{x}^{2} x=0 \\
\ddot{y}+n_{y}{ }^{2} y=0 \\
\ldots \ldots
\end{array}\right.
$$

arising from (6) (p. 281) by omission tof the terms $\frac{\partial R}{\partial x}$, $\frac{\partial R}{\partial y}, \ldots . .$. according to the method of Hamilton-Jacobi, we may arrive at

$$
\left.\begin{array}{c}
x=\frac{\sqrt{\alpha_{x}}}{n_{x}} \cos \left(n_{x} t+2 n_{x} \beta_{x}\right), \\
y=\frac{\sqrt{\alpha_{y}}}{n_{y}} \cos \left(n_{y} t+2 n_{y} \beta_{y}\right), \\
\ldots \ldots \ldots
\end{array}\right\} . . . . .
$$

We must suppose $\alpha_{x}, \alpha_{y}, \ldots \ldots$ to be of order $h^{2}$, as the amplitudes of the vibrations must be of order $h$.

When we perform the substitution of the expressions (8) in $\mathrm{R}$ we meet with two sorts of terms, namely, those which contain $t$ explicitly and those which do not contain $t$ explicitly. Only the terms of this last sort are of importance for the first approximation; the others we omit.

From a term $x^{\bar{p}} y^{\bar{q}} z^{\bar{r}} u^{\bar{s}}$ arises by the substitution only one term with cosine, which does not contain $t$ (by $\bar{p}, \bar{q}, \bar{r}, \bar{s}$ are meant the absolute values of the coefficients $p, q, r, s$ of $n_{x}, n_{y}, n_{z}, n_{\boldsymbol{u}}$ in the relation). This term is :

$$
\frac{1}{2^{\mathrm{S}-1}} \cdot \frac{\sqrt{\alpha_{x}^{\bar{p}} \alpha_{y}^{\bar{q}} \alpha_{z}^{\bar{r}} \alpha_{u}^{\bar{s}}}}{n_{x}^{\bar{p}} n_{y}^{\bar{q}} n_{z}^{\bar{r}} n_{u}^{\bar{s}}} \cos 2\left(p n_{x} \beta_{x}+q n_{y} \beta_{y}+r n_{z} \beta_{z}+s n_{u} \beta_{u}\right)
$$

where $\mathrm{S}=\bar{p}+\bar{q}+\bar{r}+\bar{s}$.

The term $\rho n_{v}{ }^{\prime} v^{2}$ appearing in $\mathrm{R}(\S 13, \mathrm{p} .281)$ gives rise to a term $\frac{\rho}{2 n_{v}^{\prime}} \alpha_{v}$. 
The terms $\mathbf{K}_{x} x^{4}, \mathrm{~K}_{y} y^{1}, \ldots \ldots, \mathrm{M}_{x x} \mathrm{~A}^{2} h^{2} x^{2}, \mathbf{M}_{y x} \mathrm{~B}^{2} h^{2} x^{2}, \ldots \ldots$ (in these latter terms we must first replace $\mathrm{A}^{2} h^{2}, \mathrm{~B}^{2} h^{2}, \ldots \ldots$ by $\left.\frac{\alpha_{x}}{n_{x}}, \frac{\alpha_{y}}{n_{y}{ }^{2}}, \ldots ..\right)$ give rise to a homogeneous quadratic function of the $\alpha^{\prime}$ 's $\chi_{2}\left(\alpha_{1}, \alpha_{2} \ldots \ldots \alpha_{m}\right)$.

$W_{\theta}$ must, however, make an exception as to the case $\mathbf{S}_{1}=2$. The terms $\mathbf{K}_{x} x^{4}, \mathbf{K}_{y} y^{4}, \ldots \ldots, \mathbf{M}_{x x} \mathrm{~A}^{2} h^{2}, \mathbf{M}_{y x} \mathrm{~B}^{2} h^{2}, \ldots \ldots$ do not give any difficulty. The term $x^{2} y^{2}$ gives, besides a term with cosine, rise to a constant term. The terms $x^{3} y$ and $x: y^{3}$ give rise resp. to terms with

$\alpha_{x} \sqrt{\alpha_{x} \alpha_{y}} \cos 2\left(n_{x} \beta_{x}-n_{y} \beta_{y}\right)$ and $\alpha_{y} \sqrt{x_{x} \alpha_{y}} \cos 2\left(n_{x} \beta_{x}-n_{y} \beta_{y}\right)$.

Therefore $\mathrm{R}$ takes in the different cases the following. form :

$$
\begin{aligned}
& 2 n_{x}-n_{y}=\rho \quad \mathrm{R}= \\
& \frac{\rho}{2 n_{y}} \alpha_{y}-\frac{d_{2}}{4 n_{x}^{2} n_{y}} \alpha_{x} \sqrt{\alpha_{y}} \\
& \cos 2\left(2 n_{x} \beta_{x}-n_{y} \beta_{y}\right) \text {. } \\
& n_{x}+n_{y}-n_{z}=\rho \quad \mathrm{R}= \\
& \frac{\rho}{2 n_{z}} \alpha_{z}-\frac{p^{\prime}}{4 n_{x} n_{y} n_{z}} \sqrt{\alpha_{x} \alpha_{y} \boldsymbol{\alpha}_{z}} \\
& \cos 2\left(n_{x} \beta_{x}+n_{y} \beta_{y}-n_{z}\right. \\
& 3 n_{x}-n_{y}=\rho \quad \mathrm{R}=\chi_{2}\left(\alpha_{x}, \alpha_{y}, \ldots \alpha_{m}\right)+\frac{\rho}{2 n_{y}} \alpha_{y}-\frac{e_{2}}{8 n_{x}^{3} n_{y}} \alpha_{x} / \alpha_{x} \alpha_{y} \\
& \cos 2\left(3 n_{x} \beta_{x}-n_{y} \beta_{y}\right) \text {. } \\
& \pm n_{x}+2 n_{y}-n_{z}=\rho \quad \mathrm{R}=\chi_{2}\left(\alpha_{x}, \alpha_{y}, \ldots \alpha_{m}\right)+\frac{\rho}{2 n_{z}} \alpha_{z}-\frac{p^{\prime}}{\delta n_{x} n_{y}{ }^{2} n_{z}} \alpha_{y} \sqrt{\alpha_{x} a} \\
& \cos 2\left( \pm n_{x} \beta_{x}+2 n_{y} \beta_{y}-n_{z} \beta_{z}\right. \\
& n_{x}+n_{y} \pm n_{z}-n_{u}=\rho \quad \mathrm{R}=\chi_{2}\left(\alpha_{x}, \alpha_{y}, \ldots \alpha_{m}\right)+\frac{\rho}{2 n_{u}} \alpha_{u}-\frac{p^{\prime}}{8 n_{x} n_{y} n_{z} n_{x}} \sqrt{\alpha_{x} \alpha} \\
& \cos 2\left(n_{x} \beta_{x}+n_{y} \beta_{y} \pm n_{z} \beta_{z} \rightarrow\right. \text { r } \\
& n_{x}-n_{y}=\rho \quad \mathrm{R}=\chi_{2}\left(\alpha_{x}, \alpha_{y}, \ldots \alpha_{m}\right)+\frac{\rho}{2 n_{y}} \alpha_{y}-\frac{f}{8 n_{x}^{2} n_{y}^{2}} \alpha_{x} \alpha_{y} \cos 4 \\
& \left.-n_{y} \beta_{y}\right)-\frac{3}{8} \sqrt{\alpha_{x} \alpha_{y}}\left(e_{2}{ }^{\prime} \alpha_{x}+e_{4}{ }^{\prime} \alpha_{y}\right) \cos 2\left(n_{x} \beta_{x}-r\right.
\end{aligned}
$$

$\S 16$. We can make now the following general observation. The $\beta$ 's appear only under the sign cosine; the coefficients of $n_{x} \beta_{x}, n_{y} \beta_{y}, \ldots$ in the expressions between brackets are the same as the coefficients in the relation (5) (p. 270). We put now:

$$
\phi \equiv 2\left(p n_{x} \beta_{x}+q n_{y} \beta_{y}+\ldots \ldots\right) \ldots . .
$$


about a Position of Equilibrium.

As

$$
\frac{\frac{\partial \mathrm{R}}{\partial \beta_{x}}}{p n_{x}}=\frac{\partial \mathrm{R}}{q \beta_{y}}=\ldots . .
$$

we have, according to (7) (p. 283):

$$
\frac{d \alpha_{x}}{p n_{x}}=\frac{d \alpha_{y}}{q n_{y}}=\ldots . . . \quad . \quad . \quad . \quad .
$$

This enables us to express the $m a$ 's by the help of one new variable $\zeta$ only; this new variable $\zeta$ we will choose in such al way that each of the members of $(10)$ is equal to $\mathrm{R}_{0}{ }^{2} h^{2} d \zeta$, where $R_{0}$ is a constant of integration of moderate value.

Now we have

$$
\alpha_{x}=p n_{x} \mathrm{R}_{0}^{2} h^{2} \zeta, \quad \alpha_{y}=q n_{y} \mathrm{R}_{0}{ }^{2} h^{2}\left(\mathrm{C}_{2}+\zeta\right), \ldots \ldots .
$$

where $\mathrm{C}_{2}, \ldots \ldots$ are $(m-1)$ constants of integration.

As in $(10)$ the sum of the denominators is zero $(\$ 13, \mathrm{p} .282)$, the sum of the numerators is also zero. Therefore

Or

$$
\frac{d \alpha_{x}}{d t}+\frac{d \alpha_{y}}{d t}+\ldots \ldots=0 .
$$

$$
\alpha_{x}+\alpha_{y}+\ldots . .=\text { constant. . . . . }
$$

In case $m=2$, then $q n_{y}{ }^{\prime}=-p n_{x}$. In this case we have

$$
\alpha_{x}=p n_{x} \mathrm{R}_{0}^{2} h^{2} \zeta, \quad \alpha_{y}=-p n_{x} \mathrm{R}_{0}{ }^{2} h^{2}(\mathrm{C}+\zeta) .
$$

Introducing $\zeta^{\prime}$ instead of $\zeta$ and $R_{0}{ }^{\prime}$ instead of $R_{0}$ in such a way that

$$
\zeta=-\mathrm{C}_{2} \zeta^{\prime}, \quad \mathrm{R}_{0}^{2}=-\frac{n_{x}}{\rho \mathrm{C}_{2}} \mathrm{R}_{0}^{\prime 2}
$$

we get, after omitting the accents again :

$$
\alpha_{x}=\mathrm{R}_{0}^{2} n_{x}^{2} h^{2} \zeta, \quad \alpha_{y}=\mathrm{R}_{0}^{2} n_{x}{ }^{2} h^{2}(1-\zeta) . \quad . \quad .
$$

$\S 17$. When we differentiate $\mathrm{R}$ according to the time, we get

$\frac{d \mathrm{R}}{d t}=\frac{\partial \mathrm{R}}{\partial \alpha_{x}} \cdot \frac{d \alpha_{x}}{d t}+\frac{\partial \mathrm{R}}{\partial \beta_{x}} \cdot \frac{d \beta_{x}}{d t}+\frac{\partial \mathrm{R}}{\partial \alpha_{y}} \cdot \frac{d \alpha_{y}}{d t}+\frac{\partial \mathrm{R}}{\partial \beta_{y}} \cdot \frac{d \beta_{y}}{d t}+\ldots \ldots+\frac{\partial \mathrm{R}}{\partial t}$.

From (7) (p. 283) we deduce

$$
\frac{d \mathrm{R}}{d t}=\frac{\partial \mathrm{R}}{\partial t}
$$


If now $\mathbf{R}$ does not contain $t$ explicitly (this condition is satisfied in the different cases we have to consider), then

$$
\mathrm{R}=\text { constant } . \text {. . . . . }
$$

is an integral of system (7) (p. 283).

By substitution of (11) or (13) in (14), we get a relation between $\zeta$ and $\phi$, which in the different cases has this form :

$$
\begin{aligned}
2 n_{x}-n_{y} & =\rho, \quad \zeta \sqrt{1-\zeta} \cos \phi=k+\rho^{\prime} \zeta, \quad\left(\rho^{\prime}=-\frac{\rho n_{y}}{d_{2} \mathrm{R}_{0} h}\right) . \\
n_{x}+n_{y}-n_{z} & =\rho, \quad \sqrt{\zeta\left(\zeta-\mathrm{C}_{2}\right)\left(\mathrm{C}_{3}-\zeta\right)} \cos \phi=k+\rho^{\prime} \zeta
\end{aligned}
$$

$$
\left(\rho^{\prime}=-\frac{2 \sqrt{n_{x} n_{y} n_{x} \rho}}{\mathrm{R}_{0} p^{\prime} h}\right)
$$

$3 n_{x}-n_{y}=\rho, \quad \zeta \sqrt{ } \zeta(1-\zeta) \cos \phi=p \zeta^{2}+q \zeta+r$.

$\pm n_{x}+2 n_{y}-n_{z}=\rho, \quad \zeta \sqrt{\left(\mathrm{C}_{1}-\zeta\right)\left(\mathrm{C}_{3}-\zeta\right)} \cos \phi=p \zeta^{2}+q \zeta+r$.

$n_{x} \pm n_{y}+n_{z}-n_{u}=p, \quad \sqrt{\zeta}\left(\mathrm{C}_{1}-\zeta\right)\left(\mathrm{C}_{2}-\zeta\right)\left(\mathrm{C}_{3} \pm \zeta\right) \cos \phi=p \zeta^{2}+q \zeta+r$.

$n_{x}-n_{y}=\rho, \quad \zeta(1-\zeta) \cos ^{2} \phi+l \sqrt{\zeta(1-\zeta)} \cos \phi=p \zeta^{2}+q \zeta+r$.

$\S 18$. When, in the first equation of system $(7)(\$ 14)$,

$$
\frac{d \alpha_{x}}{d t}=\frac{\partial \mathrm{R}}{\partial \beta_{x}}
$$

we replace the $\alpha$ 's by the expressions (11) or (13) of $\S 16$, then according to the form of $R(\S 15)$, the equation takes this form

$$
\frac{d \zeta}{d t}=f(\zeta) \sin \phi . \quad \text {. . . . . . }
$$

This regards the cases $S_{1}=3$ and $S_{1}=4$; for each of the relations $f(\xi)$ has another form.

In the case $S_{1}=2$ it takes this form :

$$
\frac{d \zeta}{d t}=f_{1}(\zeta) \sin 2 \phi+f_{2}(\zeta) \sin \phi . \quad \text {. . . }
$$

If now we eliminate $\phi$ between (14) and (15) or (16), then $\frac{d \zeta}{d t}$ is known as a function of $\zeta$, and $\zeta$ may be found as a function of $t$; from (11) or (13) we may find the $\alpha$ 's, and from (14) $\phi$. Thereupon $\frac{d \beta_{x}}{d t}, \frac{d \beta_{y}}{d t}$, in (7) (p. 283) are known as functions of $t$, and $\beta_{x}, \beta_{y}, \ldots \ldots$ may be 
found. The problem is reduced to quadratures. It is easy to see that in the cases $S_{1}=3$ and $S_{1}=4$ the coordinates with the help of elliptic functions can be expressed in terms of the time*.

$\S 19$. In $\$ 5$ (p. 275) we have found that in the case $S_{1}=3$ the coordinates which appear in the relation behave as if they were the only coordinates; the remaining coordinates behave as if no relation existed.

This is not true for the cases $S_{1}=4$ and $S_{1}=2$. In these cases the function $\mathrm{R}$ contains in general all $\alpha$ 's. So all $\beta$ 's are functions of $t$. The function $\mathrm{R}$ contains only the $\beta$ 's related to those coordinates which enter in the relation. Therefore the $\alpha$ 's related to the remaining coordinates are constants. These coordinates feel the influence of the relation only in their phase. The coordinates which appear in the relation feel also the influence of the remaining coordinates; this influence consists in a modification of the frequencies.

$$
\begin{aligned}
& \S 20 . \text { The expressions (15) and (16) for } \frac{d \zeta}{d t} \text { show us that } \\
& \left.\frac{d \zeta}{d t} \text { (and, according to (11), also } \frac{d \alpha_{x}}{d t}, \frac{d \alpha_{y}}{d t} \ldots . .\right) \text { are zero }
\end{aligned}
$$
when $\sin \phi=0$. So in general the $\alpha$ 's will vary periodically between two limits. The extreme values of the $\alpha$ 's will be known when the values of $\zeta$ are known for which $\sin \phi=0$. The equation, from which these values of $\zeta$ are found, is obtained by putting in (14) $\cos \phi$ equal to \pm 1 . This equation is in the case $S_{1}=3$ of the third, in the cases $S_{1}=4$ and $S_{1}=2$ of the fourth degree. In the case $S_{1}=2, \frac{d \zeta}{d t}$ may also become zero for $\cos \phi=-\frac{f_{2}(\zeta)}{2 f_{1}^{\prime}(\zeta)}$.

$\S 21$. We may remark now that the motion of the mechanism, in case an approximate relation between the principal frequencies exists, is wholly different from the general case in which no relation exists. In the latter case at first approximation the principal coordinates are independent of one another; each principal coordinate performs an harmonic vibration of a constant amplitude ; in order to take into account the terms of higher order we have but to add to the motion of each principal coordinate motions of a very small amplitude (if compared with the principal vibration itself). In

* In mr dissertation (Amsterdam, 1910) these calculations have been erec'ited for the relation $2 n_{x}-n_{y}=\rho$. 
the former case the principal coordinates are no longer independent of each other; the amplitudes of the principal vibrations vary with the time; so the quantities of energy belonging to each of the coordinates vary also with the time; $(12$, p. 285) expresses that the whole of the energy is constant.

When a relation exists, then the form of motion of the mechanism will constantly alter, because the $\alpha$ 's and the $\beta$ 's vary with the time. However, after a certain lapse of time the configuration will be the same as before. In order to investigate the forms of motion, which will successively occur, it will be sufficient to discuss the relation between $\zeta$ and $\phi$. To this aim we shall represent this relation in polar coordinates with $\phi$ as polar angle, $\zeta$ (or $\sqrt{\zeta}$ or $\sqrt{\overline{1}-\zeta}$ ) as radius vector. The curves are symmetrical with respect to the origin of angles. The form of the curves changes with the value of the coefficients in the second member of the equation. The intersecting points with the origin of angles are given by $\sin \phi=0$. Now there are, letting alone the special cases, two different cases :

$1^{\circ}$. For the extreme values of $\zeta, \phi$ has the same value; then $\phi$ varies between two limits. Case of libration.

$2^{\circ}$. For the extreme values of $\zeta, \phi$ is one time 0 , one time $\pi ; \phi$ takes all values. General case.

It is clear that there are two special cases. The one is to be considered as the limit case of the libration; the extreme values of $\zeta$ have coincided ; $\sin \phi$ (or $\cos \phi$ ) is zero invariably. The form of motion does not alter. Periodic case.

To a same value of the coefficients two closed curves may correspond. For a special value of the coefficients these curves may have a common point. Then the motion approaches asymptotically to the form of motion indicated by this point. Asymptotic case.

$\$ 22$. We shall apply this to the different cases.

$$
\mathrm{S}_{1}=3 \text {. }
$$

$2 n_{x}-n_{y}=\rho$. We suppose first $\rho=0$; then we have the case of the strict relation. The relation between $\zeta$ and $\phi$ runs :

$$
\zeta \sqrt{1-\zeta} \cos \phi=k \quad(\sqrt{1-\zeta} \text { we take as radius vector }) \text {. }
$$

For $k>0$ the $\zeta-\phi$ curves lie to the right of the straight line $\phi=\frac{\pi}{2}$, for $k<0$ to the left of it ; $k=0$ furnishes degeneration into the straight line $\phi=\frac{\pi}{2}$, the point $\zeta=1$ and 
the circle $\zeta=0$. By the maximal positive and negative value of $k\left(k= \pm \frac{2}{9} \sqrt{3}\right)$ the curve has contracted into an isolated point. So we have in general the case of libration, for $k=0$ the asymptotic, and for $k= \pm \frac{2}{9} \sqrt{3}$ the periodic form of motion. The general form of motion does not occur. (Fig. 1, PI. VII.)

Now we suppose $\rho \neq 0$. Then

$$
\zeta \sqrt{1-\zeta} \cos \phi=k+\rho^{\prime} \zeta .
$$

We investigate the site and shape of the $\zeta-\phi$ curves for positive values of $\rho^{\prime}$ and for all possible values of $k$.

For $k=0$ there is degeneration into the circle $\zeta=0$, and a straight line normal to the origin of the angles at a distance $\rho^{\prime}$ from pole $O$ (the degeneration points to an asymptotic form of motion).

We have two cases now : $\rho^{\prime}<1$ and $\rho^{\prime}>1$.

$\rho^{\prime}<1$. Let us now investigate the shape of the curves for different values of $k$. For $k>0$ they lie to the right of the straight line just mentioned (case of libration); for increasing value of $k$ they contract more and more until for the maximal value of $k$, belonging to a certain value of $\rho^{\prime}$, we get an isolated point (periodic form of motion). If $-\rho^{\prime}<k<0$ the curves surround point $O$ (general form of motion); if $k=-\rho^{\prime}$ we have a curve through $O$, for $k<-\rho^{\prime}$ they lie to the left of $O$ (case of libration); for the minimal value of $k$ we again get an isolated point (periodic form of motion). (Fig. 2.)

For increasing values of $\rho^{\prime}$ the straight line separating the domains $k>0$ and $k<0$ moves to the right. The doinain $k>0$ (the domain of the former case of libration) becomes smaller and vanishes for $\rho^{\prime}=1$. For $\rho^{\prime}>1$ we therefore bave curves surrounding $O$ and curves to the left of $O$ only. When $\rho^{\prime}$ increases still more the remaining isolated point approaches to $O$ (the domain of the latter case of libration becomes smaller) and the curves farther from $O$ approach to circles. 'The general form of motion becomes preponderant more and more.

$\S 23 . n_{1}+n_{2}-n_{3}=\rho$. We take $\zeta$ as radius vector. We take first the case of the strict relation. Therefore :

$$
\sqrt{\zeta\left(\zeta-\mathrm{C}_{2}\right)\left(\mathrm{C}_{3}-\zeta\right)} \cos \phi=k \text {. }
$$

The $\zeta-\phi$ curves remain to the right or to the left of $O$ according as $k$ is positive or negative (in general we have the case of libration). For the maximal value of $k^{2}$ belonging 
to given values of $\mathrm{C}_{2}$ and $\mathrm{C}_{3}$ the curves have contracted into an isolated point (periodic form of motion). For $k=0$ degeneration takes place into the point $\zeta=0$, the circles $\zeta=\mathrm{C}_{2}$ and $\zeta=\mathrm{C}_{3}$ and the straight line $\cos \phi=0$ (asymptotic form of motion). For $\mathrm{C}_{2}=\mathrm{C}_{3}$ then of necessity $k=0$. (Fig. 3.)

Now we suppose $\rho \neq 0$. Therefore

$$
\sqrt{\zeta\left(\zeta-\mathrm{C}_{2}\right)\left(\mathrm{C}_{3}-\zeta\right)} \cos \phi=\rho^{\prime}(k-\zeta) \text {. }
$$

We may imagine $\rho^{\prime}$ to be pasitive. We give to $\mathrm{C}_{2}$ and $\mathrm{C}_{3}$ constant values and we find for a certain value of $\rho^{\prime}$ the forms of the curves satisfying the different possible values of $k$. We then see how this system of curves varies when $\rho^{\prime}$ passes throngh all values from very little to very large.

For every value of $\rho^{\prime}$ three cases can be distinguished:

$k>\mathrm{C}_{3}$. As $\zeta$ remains smaller than $\mathrm{C}_{3}$, the second member, so also $\cos \phi$, remains positive. Curves on the right of $O$ (Case of libration).

$k<C_{2}$. Gurves on the left of $O$ (Case of libration).

$\mathrm{C}_{2}<k<\mathrm{C}_{3}$. The second member, therefore also $\cos \phi$, becomes zero for $\zeta=k$. Curves which surround $O$ (General form of motion).

The domains of the plane occupied by these different kinds of curves are bounded by the curves which correspond to $k=\mathrm{C}_{3}$ and $k=\mathrm{C}_{2}$. For these values of $k$ a degeneration takes place.

For $k=\mathrm{C}_{2}$ in

$$
\zeta=\mathrm{C}_{2} \text { and } \sqrt{\zeta\left(\mathrm{C}_{3}-\zeta\right)} \cos \phi=-\rho^{\prime} \sqrt{\zeta-\mathrm{C}_{2}} .
$$

The latter curve lies on the left of $O$, it begins and ends itr the points: $\zeta=\mathrm{C}_{2}, \phi= \pm \frac{\pi}{2}$.

For $k=\mathrm{C}_{3}$ in

$$
\zeta=\mathrm{C}_{3} \text { and } \sqrt{\zeta\left(\zeta-\mathrm{C}_{2}\right)} \cos \phi=\rho^{\prime} \sqrt{\mathrm{U}_{3}-\zeta} \text {. }
$$

The latter curve lies on the right of $\mathrm{O}$; it begins and ends in the points: $\zeta=\mathrm{C}_{\hat{3}}, \phi= \pm \frac{\pi}{2}$.

The degenerated curves point to asymptotic forms of motion.

To investigate how the system of curves varies when $\rho^{\prime}$ is changed, it is sufficient to investigate the variation of the degenerated curves. The result is, that the domain of the 
curves surrounding $O$ (general form of motion) is very small for small values of $\rho^{\prime}$ and it extends according as $\rho^{\prime}$ increases, so that those curves are most important for great values of $\rho^{\prime}$. Furthermore, we notice that according as $\rho$ increases the curves surrounding $O$ approach to circles with $O$ as centre.

For each value of $\rho^{\prime}$ we get for the maximal and minimal value of $k$ an isolated point on the axis of the angles (periodic form of motion).

Fig. 4 gives some curves for a rather small value of $\rho^{\prime}$, fig. 5 for a rather great value of $\rho^{\prime}$; the -..... lines indicate the degenerated curves.

$$
\mathrm{S}_{1}=4 \text {. }
$$

$\$ 24.3 n_{x}-n_{y}=\rho$. The relation between $\zeta$ and $\phi$ runs :

$$
\zeta \sqrt{\zeta(1-\zeta)} \cos \phi=p \zeta^{2}+q \zeta+r
$$

In fig. 6 the different possibilities have heen represented, $\zeta$ is taken as radius vector. The general case is indicated by a continuous line, the case of libration too. In the periodic case $\sin \phi=0$, and in the asymptotic case the form of motion approaches to $\sin \phi=0$.

$$
\frac{n_{x}+2 n_{y}-n_{z}=\rho,}{-n_{x}+2 n_{y}-n_{z}=\rho}, \frac{n_{x}+n_{y}+n_{z}-n_{u}=\rho,}{n_{r}+n_{y}-n_{z}-n_{u}=\rho .} .
$$

The relations between $\zeta$ and $\phi$ are to be found in $\S 17$. In case of the first relation $\zeta$ remains between 0 and the smaller of $\mathrm{C}_{1}$ and $\mathrm{C}_{2}$, in case of the second relation ketween 0 and the smaller of $C_{1}$ and $C_{3}$, in case of the third relation between 0 and the smallest of $C_{1}, C_{2}$, and $C_{3}$, in case of the fourth between 0 and the smaller of $\mathrm{C}_{1}$ and $\mathrm{C}_{2}$. For the rest the $\zeta-\phi$ curves are of quite the same kind as in the case of the relation $3 n_{x}-n_{y}=\rho$.

$$
\mathrm{S}_{1}=2 \text {. }
$$

$\S 25$. We shall restrict ourselves to the case $l=0, p=-q$. Then the relation between $\zeta$ and $\phi(\S 17)$ runs :

$$
\zeta(1-\zeta) \cos ^{2} \phi=q \zeta(1-\zeta)+r .
$$

In figs. 7-13 (Pl. VII.) $\zeta$ is taken as radius vector. Every one of these figures corresponds to a certain value of $q$; in each of the figures the curves correspond to different values of $r$. 
Fig. 7. $q=-\infty$. $r=-\frac{1}{4} q$. Circle $\zeta=\frac{1}{2}$.

$-\frac{1}{4} q<r<0$. Two circles with $O$ as centre.

$r=0$. Circle $\zeta=1$, isolated point $\zeta=0$.

Fig. 8. $-\infty<q<0$.

$r=-\frac{1}{4}(q-1)$. Isolated points $\zeta=\frac{1}{2}, \sin \phi=0$.

$-\frac{1}{4} q<r<-\frac{1}{4}(q-1)$. A curve to the right and a curve to the left of $O$.

$r=-\frac{1}{4} q$. Curve with double points in $\zeta=\frac{1}{2}$, $\cos \phi=0$.

$0<r<-\frac{1}{4} q$. Two curves surrounding 0 .

$r=0$. Circle $\zeta=1$, isolated point $\zeta=0$.

Fig. 9. $q=0$.

$r=\frac{1}{4}$. Isolated points $\zeta=\frac{1}{2}, \sin \phi=0$.

$0<r<\frac{1}{4}$. A curve to the right and a curve to the left of $O$.

$r=0$. Circle $\zeta=1$, isolated point $\zeta=0$.

Fig. 10. $0<q<1$.

$r=-\frac{1}{4}(q-1)$. Isolated points $\zeta=\frac{1}{2}, \sin \phi=0$.

$0<r<-\frac{1}{4}(q-1)$. A curve to the right and a curve to the left of 0 .

$r=0$. Two straight lines passing through $\mathrm{O}$.

$-\frac{1}{4} q<r<0$. A curve below and a curve above 0 .

$r=-\frac{1}{4} q$. Isolated points $\zeta=\frac{1}{2}, \cos \phi=0$.

Fig. 11. $q=1$.

$r=0$. Origin of angles, circle $\zeta=1$.

$-\frac{1}{4} q<r<0$. A curve below and a curve above 0 . $r=-\frac{1}{4} q$. Isolated points $\zeta=\frac{1}{2}, \cos \phi=0$.

Fig. 12. $1<q<\infty$.

$r=0 . \quad$ Isolated point $\zeta=0$, circle $\zeta=1$.

$-\frac{1}{4}(q-1)<r<0$. Two curves surrounding $O$.

$r=-\frac{1}{4}(q-1)$. Curve with double points in $\zeta=\frac{1}{2}$, $\sin \phi=0$.

$-\frac{1}{4} q<r<-\frac{1}{4}(q-1)$. A curve below and a curve above 0 . $r=-\frac{1}{4} q$. Isolated points $\zeta=\frac{1}{2}, \cos \phi=0$.

Fig. 13. $q=\infty$.

The same as for $q=-\infty$. 
Geometrical representation of the motion of the mechanism.

We may obtain a geometrical representation of the motion by making use of a representative point, which moves in a space of 2,3 , or 4 dimensions, if the mechanism we have in view has resp, 2, 3, or 4 degrees of freedom. From what is said, $\S 5$ and $\S 19$, it follows that it is not necessary to consider mechanisms with more than 4 degrees of freedom.

\section{Mechanism with two degrees of freedom.}

\$26. For an arbitrary mechanism with two degrees of freedom we may regard as a representative point the horizontal projection of a material point which moves without friction yet under the influence of gravitation on a given surface in the vicinity of its lowest point. The surface has positive curvature in the vicinity of this point $O$, plane $X Y$ is the tangential plane in $\mathrm{O}$, and the $\mathrm{XZ}$ - and $\mathrm{YZ}$-planes are the principal sections of the surface in that point, whilst the Z-axis is supposed positive upwards.

Then we have :

$$
\begin{aligned}
\mathrm{U}=g z=c_{1} x^{2} & +c_{3} y^{2}+d_{1} x^{3}+d_{2} x^{2} y+d_{3} x^{2} y^{2}+d_{4} y^{3} \\
& +e_{1} x^{4}+e_{2} x^{3} y+e_{3} x^{2} y^{2}+e_{4} x y^{3}+e_{5} y^{4}+\ldots \ldots \ldots,
\end{aligned}
$$

where

$$
c_{1}=\frac{1}{2} n_{x}^{2}=\frac{g}{2 \vec{R}_{x}^{-}} ; \quad c_{3}=\frac{1}{2} n_{y}{ }^{2}=\underset{2}{2 \overrightarrow{\mathrm{R}}_{y}},
$$

$\mathbf{R}_{x}$ and $\mathrm{R}_{y}$ being the principal radii of curvature of the surface in 0 .

The expression for the kinetic energy takes the form :

$$
\mathrm{T}=\frac{1}{2}\left\{\dot{x}^{2}+\dot{y}^{2}+\left(\frac{\partial z}{\partial x} \dot{x}+\frac{\partial z}{\partial y} \dot{y}\right)^{2}\right\} .
$$

$\S 27$. The motion of the representative point is given by. $(8$, p. $283 ; 13$, p. 285) :

$$
\begin{aligned}
& x=\mathrm{R}_{0} h \sqrt{\zeta} \cos \left(n_{x} t+2 n_{x} \beta_{x}\right), \\
& y=\frac{1}{\gamma} \mathrm{R}_{0} h \sqrt{1-\zeta} \cos \left(\gamma n_{x} t+2 \gamma n_{x} \beta_{y}\right)
\end{aligned}
$$

where, according to $\S 17$, a relation exists between $\zeta$ and $\phi$ :

$$
f(\zeta, \phi)=0 \text {. }
$$

$\phi$ is written instead of $2 \gamma n_{x}\left(\beta_{x}-\beta_{y}\right)$.

$\zeta, \beta_{1}$, and $\beta_{2}$ have for every moment a definite value; these values determine a certain Lissajous curve. This curve we 
shall call the osculating curve for the moment indicated, which name is in use in the theory of disturbances (see, among others, H. Poincaré, Lecons de Mécanique céleste, vol. i. p. 90). As $\zeta, \beta_{1}$, and $\beta_{2}$ vary slowly, the osculating curve will also vary; we shall examine the system of Lissajous curves described.

We take a new unit of length, having $R_{0} h$ old units; then the amplitudes become resp. $\sqrt{\zeta}$ and $\frac{1}{\gamma} \sqrt{1-\zeta}$. We see immediately that the vertices of the rectangles in which the Lissajous curves are described lie on the circumference of an ellipse with its great axis along the $\mathrm{X}$-axis; the half of the great axis is the new unit of length; the half of the small axis is the $\gamma$ th part of the new unit of length.

We may for each osculating curve chooss the origin of time in such a way that for $t=0$ the moving point has its greatest deviation to the right. The difference in phase is $2_{\gamma n_{x}}\left(\beta_{x}-\beta_{y}\right)=\phi$, when the phase is calculated from this same moment. Taking at last a new unit of time, we may write the equations in this form:

$$
\left.\begin{array}{l}
t=\sqrt{\zeta} \cos t \\
y=\frac{1}{\gamma} \sqrt{1-\zeta} \cos (\gamma t-\phi)
\end{array}\right\} \cdot . .
$$

Every osculating curve is described in $2 \pi$ new units of time.

The relation between $\zeta$ and $\phi$ has the form :

$$
\zeta^{\frac{\gamma}{2}}(1-\zeta)^{\frac{1}{2}} \cos \phi=f(\zeta), \quad . \quad . \quad . \quad .
$$

where $f(\zeta)$ has for each of the cases $\gamma=1 \quad\left(S_{1}=2\right), \gamma=2$ $\left(\mathrm{S}_{1}=3\right)$, and $\gamma=3\left(\mathrm{~S}_{1}=4\right)$ another form, namely :

$$
\begin{array}{ll}
\gamma=2 & f^{\prime}(\zeta) \equiv k+\rho^{\prime} \zeta . \\
\gamma=3 & f(\zeta) \equiv p \zeta^{2}+q \zeta+r . \\
\gamma=1 & f^{\prime}(\zeta) \equiv-\frac{1}{2} l \pm \sqrt{p \zeta^{2}+q \zeta+r+\frac{1}{4}} l^{2} .
\end{array}
$$

$\$ 28$. The shape of a Lissajous curve depends on the ratio of the periods, the ratio of the amplitudes, and the difference in phase. The shape of the curves for the ratio $\frac{1}{1}, \frac{1}{2}, \frac{1}{3}$ of the periods is well known for arbitrary values of $\phi$ as well as for the particular values of $\phi$ for which $\sin \phi=0$ or $\cos \phi=0$. For $\sin \phi=0$ we obtain the curves which are described in both directions alternately; we shall call them Lissajous double curves. That the condition $\sin \phi=0$ is 
necessary for such a curve may be proved as follows : In a double curve $\cos t$ and $\cos (\gamma t-\dot{\phi})$ take the same value for two different values of $t$; for this value of $t \sin t$ and $\sin (\gamma t-\phi)$ have a different sign. Let $t_{0}$ be one of the values for $t$, then the other must be $t^{\prime}=2 r_{1} \pi-\tau_{0}$, and in the same time $\gamma t^{\prime}-\phi=2 r_{2} \pi-\gamma t_{0}+\phi$, where $r_{1}$ and $r_{2}$ are integers. From this it follows that $\phi=\left(r_{1} \gamma-r_{2}\right) \pi$; therefore $\sin \phi=0$. That the condition $\sin \phi=0$ is sufficient for such a curve follows directly by substituting $\phi=r \pi$ in the expression for $y$.

For $\cos \phi=0$ the $\mathrm{X}$-axis and $\mathrm{Y}$-axis are lines of symmetry.

Now in $\$ 20$ we have found that in general $\zeta$ varies periodically between two extremes; the vertices of the rectangles, in which the osculating curves are described, move to and fro along the ellipse between two limits; at the same time $\phi$ varies. In the cases $S_{1}=3$ and $S_{1}=4 \frac{d \zeta}{d t}=0$ for $\sin \phi=0(\$ 20$, p. 287); so in the extreme rectangles Lissajous double curves are described. However, in the case $S_{1}=2$ $\frac{d \zeta}{d t}=0$ for $\sin \phi=0$ or (in case $l=0$ in $\S 17$ ) for $\cos \phi=0$ $(\S 20$, p. 287); so here it may occur that in one of the extreme rectangles or in botle a symmetric Lissajous curve is described.

We have seen $(\S 21)$ that there are two different special cases :

$1^{\circ}$. The periodic case. $\zeta$ remains constant. In the cases $\gamma=2$ and $\gamma=3 \sin \phi=0$; the same curve, a Lissajous double curve, is continually deseribed. In the case $\gamma=1 \sin \phi=0$ or $\cos \phi=0$; a double curve or a symmetric curve is continually described.

$2^{\circ}$. The asymptotic case. To the form of motion, just described, the motion approaches asymptotically.

\section{Envelope of the system of Lissajous curves *.}

$\S 29$. In order to find the envelope of the system of curves

$$
x=\mathrm{F}_{1}(\zeta, \phi, t), \quad y=\mathrm{F}_{2}(\zeta, \phi, t), \quad \phi=\chi(\zeta),
$$

we may regard that envelope as the locus of the intersecting points of a certain curve, given by a certain value of $\zeta$, with

* In my prize essay was only discussed the envelope for system (17, $\$ 27)$. Prof. Korteweg, being one of the examiners, proved that some of my results remained true for the more general system (19). Of his observations I have made use in the following pages. 
the curve corresponding to a value $\zeta+\Delta \zeta$ for $\zeta$. So we have to eliminate $\Delta \zeta, \Delta \phi$, and $\Delta t$ between :

$$
\begin{aligned}
\frac{\partial x}{\partial \zeta} \Delta \zeta+\frac{\partial x}{\partial \phi} \Delta \phi+\frac{\partial x}{\partial t} \Delta t & =0, \\
\frac{\partial y}{\partial \zeta} \Delta \zeta+\frac{\partial y}{\partial \phi} \Delta \phi+\frac{\partial y}{\partial t} \Delta t & =0, \\
\Delta \phi & =\chi^{\prime}(\zeta) \Delta \zeta .
\end{aligned}
$$

The result is the following equation :

$$
\frac{\partial y}{\partial t} \cdot \frac{\partial x}{\partial \zeta}-\frac{\partial x}{\partial t} \cdot \frac{\partial y}{\partial \zeta}+\left[\frac{\partial y}{\partial t} \cdot \frac{\partial x}{\partial \phi}-\frac{\partial x}{\partial t} \cdot \frac{\partial y}{\partial \phi}\right] \cdot \frac{d \phi}{d \zeta}=0 .
$$

If we eliminate $\zeta, \phi$, and $t$ between this equation and the equations written down at the beginning of this section, the result is the equation of the envelope.

$\S 30$. We start from a system of Lissajous curves which is more general than the system (17) of $\S 27$, namely

$$
x=f_{1}(\zeta) \cos t, \quad y=f_{2}(\zeta) \cos (\gamma t-\phi), \quad \cos \phi=\psi(\zeta) .
$$

By substitution of these expressions for $x$ and $y$, the equation of the envelope takes this form :

$\gamma f_{2}(\zeta) f_{1}^{\prime}(\zeta) \cos t \sin (\gamma t-\phi) \sin \phi-f_{1}(\zeta) f_{2}{ }^{\prime}(\zeta) \sin t \cos (\gamma t-\phi) \sin \phi$

$$
+f_{1}(\zeta) f_{2}(\zeta) \sin t \sin (\gamma t-\phi) \psi^{\prime}(\zeta)=0
$$

$\S 31$. This equation is satisfied by $\sin \phi=0, \sin t=0$. Now the double curves correspond to $\sin \phi=0$; the ends of these curves (meant in a dynamical sense) correspond to $\sin t=0$. Therefore :

The ends of the double curves lie on the envelope.

The tangential line of the envelope in a certain point coincides with the tangential line of the osculating curve, which in the point considered is enveloped. Therefore we have for the envelope :

$$
\frac{d y}{d x}=\frac{\gamma f_{2}(\zeta) \sin (\gamma t-\phi)}{f_{1}(\zeta) \sin t} . \quad . \quad . \quad .
$$

So we see that $\frac{d y}{d x}$ takes the indefinite form for $\sin t=0$. (this involves $\sin \gamma t=0$ ) and $\sin \phi=0$, in other words, for the ends of the double curves. We have now to investigate in what manner the ends of the double curves behave in the envelope, for instance, the point $\phi=0, t=0$. We have for this point :

$$
\frac{d y}{d x}=\gamma_{f_{1}(\zeta)}^{f_{2}(\zeta)}\left(\gamma-\frac{d \phi}{d t}\right)
$$


In order to determine $\frac{d \phi}{d t}$ we have to differentiate $(20)$ twice ; in the result we substitute $\sin t=0, \cos t=1, \sin \phi=0$, $\cos \phi=1, \sin (\gamma t-\phi)=0, \cos \left(\gamma^{t}-\phi\right)=1$. When we get :

$\gamma f_{2}(\zeta) f_{1}^{\prime}(\zeta) \frac{d \phi}{d t}\left(\gamma-\frac{d \phi}{d t}\right)-f_{1}(\zeta) f_{2}^{\prime}(\zeta) \frac{d \phi}{d t}+f_{1}^{\prime}(\zeta) f_{2}(\zeta)\left(\gamma-\frac{d \phi}{d t}\right) \psi^{\prime}(\zeta)=0$

Writing $\gamma-\left(\gamma-\frac{d \phi}{d t}\right)$ instead of $\frac{d \phi}{d t}$, where it appears as a factor, we get a quadratic equation in $\gamma-\frac{d \phi}{d t}$, namely:

$$
\begin{aligned}
& -\gamma f_{2}(\zeta) f_{1}^{\prime}(\zeta)\left(\gamma-\frac{d \phi}{d t}\right)^{2}+\left\{\gamma^{2} f_{2}(\zeta) f_{1}^{\prime}(\zeta)+f_{1}(\zeta) f_{2}^{\prime}(\zeta)\right. \\
& \left.+f_{1}(\zeta) f_{2}(\zeta) \psi^{\prime}(\zeta)\right\}\left(\gamma-\frac{d \phi}{d t}\right)-\gamma f_{1}(\zeta) f_{2}^{\prime}(\zeta)=0 .
\end{aligned}
$$

Therefore the ends of the double curves are double points in the envelope.

When $\left(\frac{d y}{d x}\right)_{1}$ and $\left(\frac{d y}{d x}\right)_{2}$ are the values of $\frac{d y}{d x}$ in the double point, then

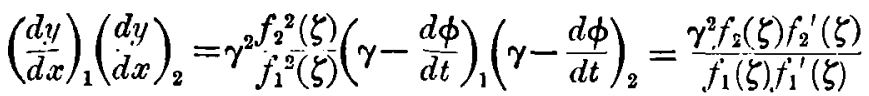

$$
\begin{aligned}
& =\gamma^{2} \frac{d\left[f_{2}^{2}(\zeta)\right]}{d\left[f_{1}^{2}(\zeta)\right]}=-1+\frac{d\left[f_{1}^{2}(\zeta)+\gamma^{2} f_{2}^{2}(\zeta)\right]}{d\left[f_{1}^{2}(\zeta)\right]} .
\end{aligned}
$$

In case

$$
f_{1}^{2}(\zeta)+\gamma^{2} f_{2}^{2}(\zeta)=\text { constant }
$$

the product $\left(\frac{d y}{d x}\right)_{1} \cdot\left(\frac{d y}{d x}\right)_{2}=-1$, i.e. the branches are in this case rectangular.

As $\frac{1}{2} f_{1}^{2}(\zeta)$ is the value of the energy of the $\mathrm{X}$-vibration, $\frac{1}{2} \gamma^{2} f_{2}{ }^{2}(\zeta)$ the value of the energy of the $Y$-vibration, the condition implies the conservation of energy.

We have found now :-

The ends (meant in a dynamical sense) of the double curves of sristem (19) appear as double points in the envelope. If the energy of the motion is constant, then the branches in the double points are rectangular.

In case of the motion, indicated by $(17, \S 27)$, the energy is constant; so for the different systems of curves we shall

Phil. Mag. S. 6. Vol. 26. No. 152. Aug. 1913. X 
have to consider the branches in the double point are always rectangular. In this case

(20) passes into

$$
f_{\mathrm{l}}(\zeta)=\sqrt{\zeta}, f_{2}(\zeta)=\frac{1}{\gamma} \sqrt{1-\zeta}, \psi(\zeta)=\frac{f(\zeta)}{\sqrt{\zeta^{\gamma}}(1-\overline{1})}
$$

$\gamma(1-\zeta) \sin (\gamma t-\phi) \sin (t-\phi)-\zeta \sin \gamma t \sin t$

$$
=2 \sqrt{\frac{1-\zeta}{\zeta^{\gamma}-2} f^{\prime}(\zeta) \sin (\gamma t-\phi) \sin t . .}
$$

$\S 32$. We now shall investigate whether the envelope possesses points where the tangential line is parallel to the $\mathrm{X}$-axis or to the $\mathrm{Y}$-axis. We suppose $f_{1}(\zeta)$ and $f_{2}(\zeta)$, the amplitudes of the vibrations, not to become infinite ; however, $f_{1}^{\prime}(\zeta)$ and $f_{2}^{\prime}(\zeta)$ may become infinite.

From

$$
\frac{d y}{d x}=\frac{\gamma f_{2}(\zeta) \sin (\gamma t-\phi)}{f_{1}^{\prime}(\zeta) \sin t}
$$

it follows that $\frac{d y}{d x}=0$, when $f_{2}(\zeta)=0$, or $\sin (\gamma t-\phi)=0$, whilst $f_{1}(\zeta) \neq 0$ and $\sin t \neq 0$. If $f_{2}(\zeta)=0$, then in general $f_{1}^{\prime}(\zeta)$ or $\psi^{\prime}(\zeta)$ will not at the same time be infinite, and $f_{1}(\zeta), f_{2}^{\prime}(\zeta)$ or $\sin \phi$ will not in general he zero at the same time. Therefore we may deduce from $(20, \S 30)$ that for $f_{2}^{\prime}(\zeta)=0 \cos (\gamma t-\phi)=0$. If $\sin (\gamma t-\phi)=0$, then either $f_{1}^{\prime}(\zeta)=\infty$, or $\psi^{\prime}(\zeta)=\infty$, or $f_{1}(\zeta)=0$, or $f_{2}^{\prime}(\zeta)=0$, or $\sin \phi=0$ (according to 20). The last condition, if combined with $\sin (\gamma t-\phi)=0$, gives the summits of the double curves. For $f_{1}(\zeta)=0 \frac{d y}{d x}$ takes the indefinite form; this condition gives in general multiple points. So we may say : the tangential line to the envelope is parallel to the $\mathrm{X}$-axis

(1) at the summits of the double curves, and

(2) at the points of the X-axis given by $f_{2}(\xi)=0$, $\cos (\gamma t-\phi)=0$.

From

$$
\frac{d y}{d x}=\frac{\gamma f_{2}(\zeta) \sin (\gamma t-\phi)}{f_{1}(\zeta) \sin t}
$$

it follows that $\frac{d y}{d x}=\infty$, when $f_{1}(\zeta)=0$ or $\sin t=0$, whilst $f_{2}(\zeta) \neq 0$ and $\sin (\gamma t-\phi) \neq 0$. If $f_{1}(\zeta)=0$, then in general $\cos t=0$ (according to 20). If $\sin t=0$, then either $._{2}^{\prime}(\zeta)=\infty$, or $\psi^{\prime}(\zeta)=\infty$, or $f_{2}(\zeta)=0$, or $f_{1}^{\prime}(\zeta)=0$, or $\sin \phi=0$. The last condition in connexion with $\sin t=0$ 
would give the ends of the double curves. For $f_{2}(\zeta)=0 \frac{d y}{d x}$ takes the indefinite form; this condition gives in general multiple points.

So we have found: the only points where the tangential line to the envelope is parallel to the $\mathrm{Y}$-axis are given by $f_{1}(\zeta)=0, \cos t=0$.

$\S 33$. In several simple cases that will be discussed afterwards, we may observe that cusps may appear in the envelope. In order to examine the appearance of these points we must put $\frac{d^{2} y}{d x^{2}}=\infty$. Wo write $(21, \S 31)$ in this form :

$$
f_{1}(\zeta) \sin t \frac{d y}{d x}-\gamma f_{2}(\zeta) \sin (\gamma t-\phi)=0 \text {. }
$$

Differentiation according to $t$ gives :

$f_{1}(\zeta) \sin t \frac{d^{2} y}{d x^{2}} \cdot \frac{d x}{d t}+f_{1}^{\prime}(\zeta) \sin t \frac{d \zeta}{d t} \cdot \frac{d y}{d x}+f_{1}(\zeta) \cos t \frac{d y}{d x}-\gamma f_{2}^{\prime}(\zeta) \frac{d \zeta}{d t} \sin (\gamma t-\phi)$

$$
-\gamma^{2} f_{2}(\zeta) \cos (\gamma t-\phi)+\gamma f_{2}(\zeta) \sin (\gamma t-\phi) \frac{d \phi}{d t}=0 \text {. }
$$

We have successively to discuss these conditions : $\sin t=0, f_{1}(\zeta)=0, \frac{d x}{d t}=0, \frac{d y}{d x}=\infty, f_{1}^{\prime}(\zeta)=\infty, f_{2}^{\prime}(\zeta)=\infty, \frac{d \zeta}{d t}=\infty, \frac{d \phi}{d t}=\infty$

Sin $t=0$ gives, as we have found $\S 32$, either $f_{2}^{\prime}(\zeta)=\infty$, or $\psi^{\prime}(\zeta)=\infty$, or $f_{2}(\zeta)=0$, or $f_{1}^{\prime}(\zeta)=0$, or $\sin \phi=0$. For $\sin t=0$, combined with $f_{2}(\zeta)=0, \frac{d y}{d x}$ takes the indefinite form ; this gives in general multiple points. Sin $\phi=0$ with $\sin t=0$ would give the ends of the double curves. Therefore we have to combine $\sin t=0$ with one of the conditions $f_{2}^{\prime}(\zeta)=\infty, \psi_{1}^{\prime}(\zeta)=\infty$, or $f_{1}^{\prime}(\zeta)=0$.

In $\S 32$ we have seen that for $f_{1}(\zeta)=0 \cos t=0$ in general. The condition $\frac{d x}{d t}=0$ may be written in this form :

$$
\frac{d \zeta}{d t}=\frac{f_{1}(\zeta)}{f_{1}^{\prime}(\zeta)} \cdot \tan t
$$

$\frac{d y}{d x}=\infty$ involves either $f_{1}(\zeta)=0$ or $\sin t=0$; these conditions have been discussed already.

For $f_{1}^{\prime}(\xi)=\infty$ we have in general, according to (20), $\cos t=0$, or $\sin (\gamma t-\phi)=0$.

For $f_{2}^{\prime}(\zeta)=\infty$ we have in general, according to $(20)$, $\sin t=0$, or $\cos (\gamma t-\phi)=0$. 
In order to examine the conditions $\frac{d \zeta}{d t}=\infty$ and $\frac{d \phi}{d t}=\infty$ we write (20) in the form

$$
f(\phi, \zeta, t)=0 .
$$

From this equation and (19) it follows :

$$
\begin{aligned}
& \sin \phi \cdot \frac{d \phi}{d t}+\psi^{\prime}(\zeta) \cdot \frac{d \zeta}{d t}=0 \\
& \frac{d f}{d \phi} \cdot \frac{d \phi}{d t}+\frac{\partial f}{\partial \zeta} \cdot \frac{d \zeta}{d t}+\frac{\partial f}{\partial t}=0
\end{aligned}
$$

In general the two conditions $\frac{d \zeta}{d t}=\infty$ and $\frac{d \phi}{d t}=\infty$ coincide; they are satisfied when

$$
\begin{gathered}
\frac{\partial f}{\partial \zeta} \cdot \sin \phi-\frac{\partial f}{\partial \phi} \cdot \psi^{\prime}(\zeta)=0 . \\
\underline{2 n_{x}-n_{y}=\rho .}
\end{gathered}
$$

$\S 34$. The osculating curves are given by

whilst

$$
\left.\begin{array}{l}
x=\sqrt{\zeta} \cos t \\
y=\frac{1}{2} \sqrt{1-\zeta} \cos (2 t-\phi),
\end{array}\right\} . . .
$$

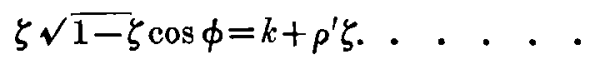

The vertices of the circumscribed rectangles lie in the circumference of the ellipse $x^{2}+4 y^{2}=1$.

The Lissajous double curves are parabolæ ; for $\phi=0$ the parabola has its opening turned upwards, for $\phi=\pi$ turned downwards.

The Lissajous curve corresponding to $\cos \phi=0$ has a double point in 0 .

The curves corresponding to an arbitrary value of $\phi$ have a node in the $\mathrm{Y}$-axis at a distance $-\frac{1}{2} \sqrt{1-\zeta} \cos \phi$ from the $\mathrm{X}$-axis.

In the case of libration the curves of the system have their nodes all at the same side of 0 ; the double curves have their opening to the same side. In the case of the general form of motion the nodes of the curves lie partly on one, partly on the other side of $\mathrm{O}$; the double curves have their openings to different sides. In the case of the periodic form of motion the same parabola is continually described. In the asymptotic case the motion approuches to a Y-vibration. 
$\S 35$. Envelope. For this case $(22, \S 31)$ takes the form :

$2(1-\zeta) \sin (2 t-\phi) \sin (t-\phi)-\zeta \sin 2 t \sin t$

$$
=2 \rho^{\prime} \sqrt{1-\zeta} \sin (2 t-\phi) \sin t .
$$

When multiplying with $\sin \phi$ we get :

$$
(1-\zeta) \sin (t-\phi)\left\{\sin ^{2} t-\sin ^{2}(t-\phi)\right\}-\zeta \sin ^{2} t \cos t \sin \phi
$$

$$
=\rho^{\prime} \sqrt{1-} \zeta \sin t\left\{\sin ^{2} t-\sin ^{2}(t-\phi)\right\} \text {. }
$$
When multiplying with $\frac{\sqrt{1-\zeta}}{\sin ^{3} t}$ and making use of (24)
we get :

$$
\begin{aligned}
-\left(\frac{\sqrt{1-\zeta} \sin (t-\phi)}{\sin t}\right)^{3} & +\left(\frac{\sqrt{1-\zeta} \sin (t-\phi)}{\sin t}\right) \\
& =k+\rho^{\prime}-\rho^{\prime}\left(\frac{\sqrt{1-\bar{\zeta}} \sin (t-\phi)}{\sin t}\right)^{2} .
\end{aligned}
$$

Putting

$$
\frac{\sqrt{1} \overline{-\zeta} \sin (t-\phi)}{\sin t}=\frac{k+\rho^{\prime} v}{v}
$$

then wo get :

$$
v^{2}(1-v)-\left(k+\rho^{\prime} v\right)^{2}=0 .
$$

Now this cubic equation has the same coefficients as the equation which serves to determine the values of $\zeta$ corresponding to sin $\phi=0$. The equation has two positive roots which we shall call $\zeta_{1}$ and $\zeta_{2}$ (we suppose $\zeta_{2}>\zeta_{1}$ ) and one negative root $-\lambda$.

So the relation between $\zeta$ and $t$ degenerates into:

$$
\begin{aligned}
& \frac{\sqrt{ } 1-\bar{\zeta} \sin (t-\phi)}{\sin t}=\frac{k+\rho^{\prime} \zeta_{1}}{\zeta_{1}}, \frac{\sqrt{1-\zeta} \sin (t-\phi)}{\sin t}=\frac{k+\rho^{\prime}}{\zeta_{2}}, \\
& \frac{\sqrt{1-\zeta} \sin (t-\phi)}{\sin t}=\frac{k-\rho^{\prime} \lambda}{-\lambda} \text {. }
\end{aligned}
$$

Now we reduce the first member of these equations as follows :-

$$
\begin{aligned}
& \underline{\sqrt{1-\zeta} \sin (t-\phi)}=\frac{\sqrt{1-\zeta} \sin (2 t-\phi) \cos t-\sqrt{1-\zeta \cos (2 t-\phi) \sin t}}{\sin t} \\
& =\frac{\sqrt{1}-\zeta \sin (2 t-\phi)}{\sqrt{\zeta} \sin t} \cdot \sqrt{\zeta} \cos t-\sqrt{ } 1-\bar{\zeta} \cos (2 t-\phi)=\frac{d y}{d x} x-2 y .
\end{aligned}
$$


So the first equation becomes :

$$
x \frac{d y}{d x}-2 y=\frac{k+\rho^{\prime} \zeta_{1}}{\zeta_{1}}
$$

Or, after integration :

$$
y=\mathrm{C} x^{2}-\frac{1}{2} \frac{k+\rho^{\prime} \zeta_{1}}{\zeta_{1}}
$$

The envelope has degenerated into three parabolæ, having the same axis ; according to $\S 31$ (p. 296) they intersect (in the ends of the double curves) under right angles, so they are confocal.

In order to determine the value of the constants $C$ we have but to recollect that one of the parabolæ passes through the points $\left(\sqrt{\zeta_{1}}, \frac{1}{2} \sqrt{1-\zeta_{1}}\right)$ and $\left(\sqrt{\zeta_{2}}, \frac{1}{2} \sqrt{1-\zeta_{2}}\right)$. Let it be the parabola

Then we have :

$$
y=\mathrm{C} x^{2}-\mathrm{C}_{1} \text {. }
$$

$$
\begin{aligned}
& \frac{1}{2} \sqrt{1-\zeta_{1}}=\mathrm{C} \zeta_{1}-\mathrm{C}_{1}, \\
& \frac{1}{2} \sqrt{1-\zeta_{2}}=\mathrm{C} \zeta_{2}-\mathrm{C}_{1} .
\end{aligned}
$$

Therefore

$$
\mathrm{C}=\frac{\sqrt{1-\zeta_{1}}-\sqrt{1-\zeta_{2}}}{2\left(\zeta_{1}-\zeta_{2}\right)}
$$

The equation from which $\zeta_{1}$ and $\zeta_{2}$ have been found runs

$$
\zeta^{2}(1-\zeta)-\left(k+\rho^{\prime} \zeta\right)^{2}=0 . . . . .
$$

From this we may deduce :

$$
\sqrt{1-\zeta_{1}}=\frac{k}{\zeta_{1}}+\rho^{\prime}, \quad \sqrt{1-\zeta_{2}}=\frac{k}{\zeta_{2}}+\rho^{\prime} .
$$

Then we get:

$$
\mathrm{C}=\frac{\frac{k}{\zeta_{1}}-\frac{k}{\zeta_{2}}}{2\left(\zeta_{1}-\zeta_{2}\right)}=-\frac{1}{2} \frac{k}{\zeta_{1} \zeta_{2}}=-\frac{1}{2} \frac{k \lambda}{\zeta_{1} \zeta_{2} \lambda} .
$$

The product of the roots of the cubic equation being $-k^{2}$,

$$
\mathrm{C}=-\frac{\lambda}{2 k} \text {. }
$$

Now we must decide, whether

$$
\mathrm{C}_{1}=\frac{k+\rho^{\prime} \zeta_{1}}{\zeta_{1}}, \frac{k+\rho^{\prime} \zeta_{2}}{\zeta_{2}}, \text { or } \frac{k-\rho^{\prime} \lambda}{-\lambda} .
$$


The parabola we have in view has its top on the outside of the ellipse that contains the vertices of the circumscribed rectangles. Now according to (25) :

$\frac{k+\rho^{\prime} \zeta_{1}}{\zeta_{1}}=\sqrt{1-\zeta_{1}}, \quad \frac{k+\rho^{\prime} \zeta_{2}}{\zeta_{2}}=\sqrt{1-\zeta_{2}}, \quad \frac{k-\rho^{\prime} \lambda}{-\lambda}=\sqrt{1+\lambda}$.

Only the last of these expressions surpasses unity. So one of the enveloping parabolæ has the equation :

$$
2 y-\frac{-\lambda}{k} x^{2}+\frac{k}{-\lambda}+\rho^{\prime}=0 .
$$

The focus of this parabola is at a distance of $-\frac{1}{2} \rho^{\prime}$ from the $X$-axis ; this tocus is at the same time the focus of the remaining enveloping parabolæ. Therefore the equations become :

$$
\left.\begin{array}{lll}
2 y-\frac{\zeta_{1}}{k} x^{2}+\frac{k}{\zeta_{1}}+\rho^{\prime}=0 & \zeta_{1} \text { parabola. } \\
2 y-\frac{\zeta_{2}}{k} x^{2}+\frac{k^{\prime}}{\bar{\zeta}_{2}}+\rho^{\prime}=0 & \zeta_{2} \quad, \\
2 y-\frac{-\lambda}{k} x^{2}+\frac{k}{-\lambda}+\rho^{\prime}=0 & \lambda \quad,
\end{array}\right\} \text {. }
$$

We must notice that the calculation is exact only in case $\phi=0$ for $\zeta=\zeta_{1}$ and $\zeta=\zeta_{2}$; if $\phi=\pi$ for one of these values of $\zeta$ or for both, then the calculation is not exact; however, it is not difficult to verify that the system of equations we arrive at is always the same.

$\S 36$. Strict relation.-In case the strict relation $n_{y}-2 n_{x}=0$ is satisfied we have $\rho^{\prime}=0$; the relation between $\zeta$ and $\phi$ runs :

$$
\zeta \sqrt{1-\zeta} \cos \phi=k \text {. }
$$

The enveloping parabolæ have their focus at $O$.

Fig. 15 gives a representation; besides the enveloping parabolæ some osculating curves, among which the double curves, have been drawn; the figure corresponds to a positive value of $k$. The enveloping parabola, which has its opening turned downwards, is the $\lambda$ parabola'; of the two remaining parabolæ, the $\zeta_{2}$ parabola has its top nearest to the $\mathrm{X}$-axis $\left(\zeta_{2}\right.$ being $\left.>\zeta_{1}\right)$.

The periodic form of motion we have for the maximal value of $k^{2}\left(\frac{4}{2}\right)$. Then $\zeta_{1}=\zeta_{2}=\frac{2}{3}, \sin \phi=0$ invariably. The motion takes place in a parabola, in which the $\zeta_{1}$ and $\zeta_{2}$ 
enveloping parabolæ have coincided (fig. 14). The X- and Y-amplitudes being resp. $\sqrt{\frac{2}{3}}$ and $\frac{1}{2} \sqrt{\frac{1}{3}}$, they have a ratio of $2 \sqrt{2}: 1$. This result was also found in another way by Lord Rayleigh *.

The asymptotic form of motion we have for $k=0$. The relation between $\zeta$ and $\phi$ degenerates into $\zeta=0, \zeta=1$, $\cos \phi=0$. In case of the asymptotic form of motion $\cos \phi=0$ invariably; the motion approaches asymptotically to a motion in the YZ-plane (fig. 16). The $\zeta_{2}$ enveloping parabola has degenerated into the $\mathrm{Y}$-axis.

\$37. Approximate relation.-The focus of the enveloping parabolæ is at a distance of $-\frac{1}{2} \rho^{\prime}$ from 0 . According to $\$ 22$ we may for a certain value of $\rho^{\prime}<1$ distinguish the following forms of motion :-

$k>0$. All is in main points the same as in the case $\rho^{\prime}=0$.

$k$ has its maximal value. The periodic form of motion corresponding to this value of $k$ differs from the periodic form of motion we had for $\rho^{\prime}=0$ only by the value of $\zeta$.

$k=0$. The $\zeta-\phi$ relation degenerates into $\zeta=0$ and $\sqrt{1-\zeta} \cos \phi=\rho$. Now according to $\S 34$ (p. 300)

$$
-\frac{1}{2} \sqrt{1-\zeta} \cos \phi
$$

is the distance of the node of an osculating curve from the $\mathrm{X}$-axis. Therefore in the asymptotic case all osculating curves have their nodes at the same point of the Y-axis, at a distance of $-\frac{1}{2} \sqrt{1-\zeta} \cos \phi=-\frac{1}{2} \rho^{\prime}$ from the $\mathrm{X}$-axis ; i. o. w. the nodes are at $B$. The $\zeta_{2}$ enveloping parabola has degenerated into the $\mathrm{Y}$-axis. For $k=0$ the $\zeta_{1}$ and $\lambda$ parabolæ exchange their place ; for a positive value of $k$ the $\lambda$ parabola has its opening turned downwards, the $\zeta_{1}$ parabola upwards; for a negative value of $k$ the tables are turned (fig. 17).

$-p^{\prime}<k<0$. The nodes of the osculaing curves lie on both sides of $O$ (fig. 18).

$k=-\rho^{\prime}$. One of the Lissajous double curves has degenerated into the $X$-axis; the $\zeta_{2}$ enveloping parabola passes through $\mathrm{O}$; the nodes of the usculating curves lie above $O$.

$k<-\rho^{\prime}$. We may get the system of curves and their envelope by turning the system we have for $k>0$ over $180^{\circ}$. $k$ has its minimal value. Periodic form of motion; $\phi=\pi$ invariably.

For $\rho^{\prime}>1$ the focus of the enveloping parabolæ lies outside the ellipse $x^{2}+4 y^{2}=1$. According to $\S 22$ we have

* Phil. Mrag. $[6]$ vol. xx. (1010). 
now only to consider $k<0$; so only the four last forms of motion are possible. For increasing values of $\rho^{\prime}$ the general form of motion $\left(-\rho^{\prime}<k<0\right)$ becomes preponderant more and more; the focus of the enveloping parabolæ moves farther ; fig. 19 shows bow the limitation approaches more and more to the rectangle of the general case where no relation exists.

$$
3 n_{x}-n_{y}=\rho .
$$

$\S 38$. The osculating curves are given by :

whilst

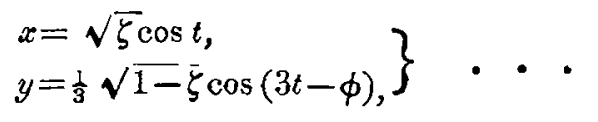

$$
\zeta \sqrt{\zeta}(\overline{1-\zeta}) \cos \phi=p \zeta^{2}+\eta \zeta+r
$$

The vertices of the circumscribed rectangles lie in the circumference of the ellipse :

$$
x^{2}+9 y^{2}=1
$$

The Lissajous curve corresponding to an arbitrary value of $\phi$ has two nodes. For $\cos \phi=0$ the nodes lie in the $\mathrm{X}$-axis on either side of $\mathrm{O}$ at distances $\bar{\nu}_{\nu}$ from $\mathrm{O}$; for an arbitrary value of $\phi$ the distance of the nodes from the $\mathrm{Y}$-axis is also $\frac{\sqrt{\zeta}}{2}$, the distance from the $\mathrm{X}$-axis is $\frac{1}{3} \sqrt{1-\zeta} \cos \phi$. One of the nodes is reached at the moments $t=\frac{\pi}{3}$ and $t=\frac{5 \pi}{3}$, the other at the moments $t=\frac{2}{3} \pi$ and $t=\frac{4}{3} \pi$.

The Lissajons double curves pass through $\mathrm{O}$.

In the case of the libration as well as in the case of the general form of motion in the extreme rectangles, double curves are described. In the former case the ends of the double curves lie two by two in opposite quadrants; in the latter case in each of the four quadrants one end lies.

§39. Enveloping curtes.-We shall restrict ourselves to the case that $p=0$ and $q=0$ in the relation between $\zeta$ and $\phi$. Then

$$
\zeta \sqrt{\zeta(1-\bar{\zeta})} \cos \phi=r . \text {. . . . . }
$$

We suppose $r$ to be positive. 
For an arbitrary value of $r$ we have the case of libration, $\cos \phi$ remaining positive. The maximal value $\left(\frac{3}{16} \sqrt{ } 3\right)$ and the minimal value of $r(0)$ give rise to the special cases resp. of the periodic and of the asymptotic form of motion.

In the case of the periodic form of motion $\zeta=\frac{3}{4}$ and $\sin \phi=0$; the same double curve is continually described, which is in the same time the envelope (fig. 20).

In the case of the asymptotic form of motion we have $\phi=\frac{\pi}{2}$. For this case (22) of p. 298 passes into:

Therefore

$$
3(1-\zeta) \cos 3 t \cos t-\zeta \sin 3 t \sin t=0 \text {. . . }
$$

$$
\zeta=\frac{3 \cos 3 t \cos t}{3 \cos 3 t \cos t+\sin 3 t \sin t} .
$$

By substitution of this in the expressions for $x$ and $y$, we find

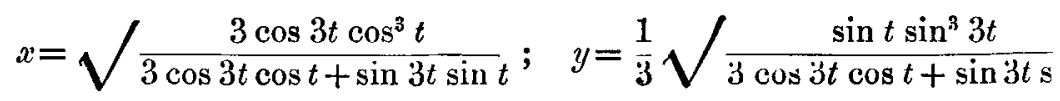

Furthermore we have :

$\frac{d y}{d x}=-\frac{\sqrt{1-\zeta} \cos 3 t}{\sqrt{\zeta} \sin t}=-\sqrt{\frac{\sin 3 t \sin t \cos ^{2} 3 t}{3 \cos 3 t \cos t \sin ^{2} t}}=-\sqrt{\frac{\sin 6 t}{3 \sin 2 t}}$.

From this it is possible to deduce the shape of the envelope. $x=0$ for $\cos 3 t=0$ and for $\cos t=0$. For $\cos 3 t=0$, $y= \pm \frac{1}{3}, \frac{d y}{d x}=0 ;$ for $\cos t=0, y= \pm \frac{1}{3}, \frac{d y}{d x}= \pm 1 . \quad y=0$ for $\sin 3 t=0$ and for $\sin t=0$. For $\sin 3 t=0, x= \pm \frac{1}{2}, \frac{d y}{d x}=0$; for $\sin t=0, x= \pm 1, \frac{d y}{d x}= \pm 1$. The enveloping curve is, besides some osculating curves, represented in fig. 22 .

Now we may put the question, what is the signification of the different parts of the envelope. A definite Lissajous curve is enveloped in different points, in other words to a definite value of $\zeta$ correspond different values of $t$ for the envelope. Let $t_{1}$ and $t_{2}$ be two of these values, then from (29) it follows

$$
\frac{\sin 3 t_{1} \sin t_{1}}{\cos 3 t_{1} \cos t_{1}}=\frac{\sin 3 t_{2} \sin t_{2}}{\cos 3 t_{2} \cos t_{2}} .
$$

After reduction this relation degenerates into

$$
\cos 2 t_{1}=\cos 2 t_{2} \quad \text { and } \cos 2 t_{1} \cos 2 t_{2}=-\frac{1}{2} .
$$

The former equation expresses only that $O$ is centre of the 
envelope. From the latter one it follows that either $\cos 2 t_{1}$ or $\cos 2 t_{2}$ is positive. Let $\cos 2 t_{1}$ be positive, then $\cos 2 t_{1}$ lies between $\frac{1}{2}$ and $1, \cos 2 t_{2}$ between $-\frac{1}{2}$ and -1 .

A Lissajous curve corresponding to $\cos \phi=0$ consists of three parts, which are separated by the two double points. For the middle part $x$ lies between $\frac{1}{2} \sqrt{ } \zeta$ and $-\frac{1}{2} \sqrt{\zeta}$; therefore $\cos t$ lies for this part between $\frac{1}{2}$ and $-\frac{1}{2}$, and $\cos 2 t$ between -1 and $-\frac{1}{2}$. For the other parts $\cos 2 t$ lies between 0 and -1 or $-\frac{1}{2}$ and +1 .

Therefore the envelope consists of a part that envelopes the middle part of the Lissajous figures and a part that envelopes the other parts.

$\S 40$. For an arbitrary value of $r$ the maximal value of $\phi$ belongs to $\zeta=3$, because $\phi$ is max., when $\cos \phi$ is min., but from $\zeta \sqrt{\zeta(1-\zeta) \cos \phi=r}$ it follows that $\cos \phi$ is min., when $\zeta^{3}(1-\zeta)$ is max., and this takes place for $\zeta=\frac{3}{4}$.

The locus of the double points of the system of curves may be determined in this way. For a definite Lissajous curve the coordinates of the double points are:

$$
x= \pm \frac{1}{2} \sqrt{\zeta}, \quad y=\mp \frac{1}{3} \sqrt{1-\zeta} \cos \phi .
$$

By eliminating $\zeta$ and $\phi$ between these two equations and the relation $\zeta \sqrt{\zeta}(\overline{1-\zeta}) \cos \phi=r$ we get :

$$
x^{3} y=-\frac{1}{24} r \text {. }
$$

On this curve lie also the summits of the double carves.

When for an arbitrary value of $r$ we wish to determine the shape of the envelope, we have to consider that this envelope has no points where the tangential line is parallel to the $\mathrm{Y}$-axis, and that the only points where the tangential line is parallel to the $\mathrm{X}$-axis are the summits of the double curves (conf. $\S 32$; here $f_{1}(\zeta)=\sqrt{\zeta}, f_{2}(\zeta)=\frac{1}{3} \sqrt{1-\zeta}$; $\zeta$ does not become 0 or 1 ).

Besides the ends of the double curves (with the direction of the tangential lines) and the summits of the double curves, some other points of the envelope may easily be found. We therefore must consider the Lissajous curve. for which $\zeta=3$.

(22) of p. 298 takes this form :

$$
3(1-\zeta) \sin (3 t-\phi) \sin (t-\phi)-\zeta \sin 3 t \sin t=0 \text {. }
$$

For $\zeta={ }_{4}^{3}$ we get

$$
\sin (3 t-\phi) \sin (t-\phi)-\sin \hat{\partial} t \sin t=0 \text {. }
$$


After reduction :

$$
t=\frac{1}{4} \phi+n \times 45^{\circ} \text {. }
$$

The eight points in which the curve we have in view is enveloped are

$$
\begin{aligned}
& x=\frac{1}{2} \sqrt{3} \cos \left(\frac{1}{4} \phi+n \times 45^{\circ}\right), \\
& y=\frac{1}{6} \cos \left(-\frac{1}{4} \phi+3 n \times 45^{\circ}\right)= \pm \frac{1}{6} \cos \left(\frac{1}{4} \phi+n \times 45^{\circ}\right) .
\end{aligned}
$$

Therefore

$$
\frac{x}{y}= \pm 3 \sqrt{ } 3 \text {. }
$$

These points are situated on the diagonals of the rectangle in which the curve is described.

Furthermore,

$$
\frac{d y}{d x}=\frac{\sqrt{1-\zeta} \sin (3 t-\phi)}{\sqrt{\zeta} \sin t}= \pm{ }_{3}^{1} \sqrt{ } 3
$$

The tangential lines in these points form with the $\mathrm{X}$-axis angles of $30^{\circ}$ or $150^{\circ}$.

In the entelope cusps appear. In order to determine the situation of these cusps, we would have to calculate $\zeta, \phi$, and $t$ from three equations, namely (28) of $\S 39,(30)$ of this section, and the third equation is $(\S 33)$ either $\frac{d \zeta}{d t}=\infty$ or $\frac{d x}{d t}=0$. However, these systems become rather intricate.

In fig. 21 the envelope with some of the Lissajous curves is represented.

$$
n_{x}-n_{y}=\rho .
$$

$\S 41$. The Lissajous curves for this case, given by

$$
\left.\begin{array}{l}
x=\sqrt{\bar{\zeta} \cos t}, \\
y=\sqrt{1-\bar{\zeta} \cos (t-\phi),}
\end{array}\right\} . \cdot
$$

are ellipses. The double curves are straight lines passing through 0 . The ellipses corresponding to $\cos \phi=0$ have their axes along the axes of coordinates. For an arbitrary value of $\phi$ the ellipse has an arbitrary shape and position.

In order to investigate the change in shape and position of the ellipse, we may write down the well-known relations which may serve for the calculation of the axes of the ellipse and the angle of inclination of the long axis with the $X$-axis. If $A$ and $B$ are half the larger and half the smaller 
axis, and if $\theta$ is the angle we have in view, then these relations become

$$
\left.\begin{array}{rl}
\frac{1}{\mathrm{~A}^{2}}+\frac{1}{\mathrm{~B}^{2}} & =\frac{1}{\zeta(1-\zeta) \sin ^{2} \phi}, \\
\frac{1}{\mathrm{~A}^{2} \mathrm{~B}^{2}} & =\frac{1}{\zeta(1-\zeta) \sin ^{2} \phi}, \\
\tan 2 \theta & =\frac{2 \sqrt{\zeta(1-\zeta)}}{1-2 \zeta} \cos \phi .
\end{array}\right\} .
$$

From the first and the second of these relations we deduce $\mathrm{A}^{2}+\mathrm{B}^{2}=1$. The vertices of an arbitrary rectangle circnnscribed to any one of the ellipses lie on the circumference of a circle with unity as radius.

In a system of ellipses there are two double curves, or there are no double curves.

$\$ 42$. Envelope. (22) of p. 298 becomes for this case $(1-\zeta) \sin ^{2}(t-\phi)-\zeta \sin ^{2} t=2 \sqrt{\zeta(1-\zeta)} f^{\prime}(\zeta) \sin (t-\phi) \sin t$.

We may write it in this form :

$$
\frac{(1-\zeta) \sin ^{2}(t-\phi)}{\zeta \sin ^{2} t}-2 f^{\prime}(\zeta) \frac{\sqrt{1-\zeta} \sin (t-\phi)}{\sqrt{\zeta} \sin t}-1=0 .
$$

According to $(21, \S 31)$ we have

Therefore

$$
\frac{d y}{d x}=\frac{\sqrt{1-\zeta} \sin (t-\phi)}{\sqrt{\zeta} \sin t} .
$$

$$
\left(\frac{d y}{d x}\right)^{2}-2 f^{\prime}(\zeta) \frac{d y}{d x}-1=0 . \quad \text {. . . }
$$

This quadratic equation in $\frac{d y}{d x}$ gives the direction of the envelope in those points in which it envelopes a same ellipse, defined by the value of $\zeta$. To each value of $\frac{d y}{d x}$ two points of the ellipse belong. The product of the roots of the equation being -1 , we can say that the tangential lines to the ellipse in the points in which it is enveloped form a rectangle.

$\S 43$. We have found already $(\S 20)$ that in the case $y=1$ $\frac{d \zeta}{d t}$ may become zero not only for $\sin \phi=0$, but also for $\cos \phi=-\frac{l}{2 \sqrt{\zeta(1-\zeta)}}$. 
There are three different cases:-

(1) In one of the extreme rectangles $\sin \phi=0$; in the other $\cos \phi=-\frac{l}{2 \sqrt{\zeta(1-\zeta)}}$. The ends of the double curve are double points of the envelope. The envelope consists of two segments.

(2) In both extreme rectangles $\sin \phi=0$. The ends of the double curves are double points of the envelope. The envelope consists of four segments.

(3) In both extreme rectangles $\cos \phi=-\frac{l}{2 \sqrt{\zeta(1-\zeta)}}$. In the system there exist no double curves; in the envelope no double points appear. The envelope consists of two closed curves, not intersecting each other. One of these curves is the outward envelope, the other the inward envelope.

$\S 44$. From the form of $f(\zeta)$ for the case $\gamma=1$, given on p. 294 , we deduce

$$
f^{\prime 2}(\zeta)+1=\frac{4 p(p+1) \zeta^{2}+4 q(p+1) \zeta+q^{2}+4 r+l^{2}}{4\left(p \zeta^{2}+q \zeta+r+\frac{1}{4} l^{2}\right)}
$$

Now from the relation (34),

$$
\frac{d y}{d x}=f^{\prime}(\zeta) \pm \sqrt{f^{\prime 2}(\zeta)+1}
$$

we come to the supposition that simple cases will occur when

$$
4 p(p+1) \zeta^{2}+4 q(p+1) \zeta+q+4 r+l^{2}
$$

is a complete square. The condition runs:

$$
4 p(p+1)\left(q^{2}+4 r+l^{2}\right)=q^{2}(p+1)^{2},
$$

which condition degenerates into

$$
p+1=0
$$

and

$$
p\left(q^{2}+4 r+l^{2}\right)=q^{2}(p+1),
$$

which may be written in this form :

$$
p\left(4 r+l^{2}\right)=q^{2}
$$

this expresses at the same time the condition that the denominator of the fraction, written down at the beginning of this section, is a complete square. In this latter case $f(\zeta)$ is a linear function of $\zeta$. Therefore we pass to the discussion of these two cases:

$$
\begin{aligned}
& p=-1 . \\
& f(\zeta) \text { is a linear function of } \zeta .
\end{aligned}
$$




\section{$\S 45$. Case $p=-1$.}

The motion in an arbitrary ellipse can be analysed in two rectangular oscillations. Taking a new system of axes of coordinates, the expressions for $x$ and $y$ hold the same form. But the values of $\zeta$ and $\phi$ for a certain ellipse will be altered in general. Considering the whole system of ellipses, we can say that the relation between $\zeta$ and $\phi$ will also be altered. The new values of $\zeta$ and $\phi$ we respectively call $\zeta^{\prime}$ and $\phi^{\prime}$.

We shall prove now that in the case supposed $(p=-1)$ it is always possible to turn the system of axes through an angle $\psi$ so that the relation between $\zeta^{\prime}$ and $\phi^{\prime}$ takes this form:

$$
\sqrt{\zeta^{\prime}\left(1-\zeta^{\prime}\right)} \cos \phi^{\prime}= \pm \sqrt{-\zeta^{\prime 2}+q \zeta^{\prime}+r^{\prime}}
$$

Or, what is the same :

$$
\sqrt{\zeta^{\prime}\left(1-\zeta^{\prime}\right)} \sin \phi^{\prime}= \pm \sqrt{m \zeta^{\prime}+n .}
$$

The new coordinates are given by

$$
x^{\prime}=x \cos \psi-y \sin \psi, \quad y^{\prime}=x \sin \psi+y \cos \psi .
$$

Therefore

$$
x^{\prime}=\sqrt{\zeta} \cos \psi \cos t-\sqrt{1-\zeta} \sin \psi \cos (t-\phi) .
$$
Then

This may be expressed in a single trigonometrical term.

$$
\zeta^{\prime}=\zeta \cos ^{2} \psi+(1-\zeta) \sin ^{2} \psi-2 \sqrt{\zeta(1-\zeta)} \cos \phi \sin \psi \cos \psi .
$$

From $(32, \S 41)$ it follows that $\zeta(1-\zeta) \sin ^{2} \phi$ is an invariant. Therefore

$$
\zeta^{\prime}\left(1-\zeta^{\prime}\right) \sin ^{2} \phi^{\prime}=\zeta(1-\zeta) \sin ^{2} \phi .
$$

If the relation between $\zeta^{\prime}$ and $\phi^{\prime}$ will take the form

$$
\zeta^{\prime}\left(1-\zeta^{\prime}\right) \sin ^{2} \phi^{\prime}=m \zeta^{\prime}+n,
$$

then it must be possible to write the relation between $\zeta$ and $\phi$ as follows :

$\zeta(1-\zeta) \sin ^{2} \phi=m\left\{\zeta \cos ^{2} \psi+(1-\zeta) \sin ^{2} \psi-2 \sqrt{\zeta(1-\zeta)} \cos \phi \sin \psi \cos \psi\right\}+n$. After reduction,

$\zeta(1-\zeta) \cos ^{2} \phi-m \sin 2 \psi \sqrt{\zeta(1-\zeta)} \cos \phi+\zeta^{2}+(m \cos 2 \psi-1) \zeta+m \sin ^{2} \psi+n=0$.

Now for $p=-1$ the relation between $\zeta$ and $\phi$ rurs

$$
\zeta(1-\zeta) \cos ^{2} \phi+l \sqrt{\zeta(1-\zeta)} \cos \phi-\left(-\zeta^{2}+q \zeta+r\right)=0 \text {. }
$$


We therefore shall have to determine $m, n$, and $\psi$ from the system

$$
\left\{\begin{aligned}
-m \sin 2 \psi & =l, \\
1-m \cos 2 \psi & =q, \\
-n-m \sin ^{2} \psi & =r .
\end{aligned}\right.
$$

From the first two equations we deduce:

$$
\begin{aligned}
& \tan 2 \psi=\frac{l}{q-1}, \\
& m^{2}=l^{2}+(q-1)^{2} .
\end{aligned}
$$

$n$ is to be found from

$$
n=-r-m \sin ^{2} \psi
$$

So it is always possible to find values of $\psi, m$, and $n$; the relation between $\zeta^{\prime}$ and $\phi^{\prime}$ may therefore be written in this forn

$$
\zeta^{\prime}\left(1-\xi^{\prime}\right) \sin ^{2} \phi^{\prime}=m \zeta^{\prime}+n .
$$

There are two values of $\psi, m$, and $n$; so there are two different systems of ellipses, each of them having its own envelope.

$\$ 46$. We suppose the turn of the axes to have taken place. Therefore we have to take the relation between $\zeta$ and $\phi$ in this form :

$$
\zeta(1-\zeta) \sin ^{2} \phi=m \zeta+n . \quad . \quad . .
$$

The equation of the envelope runs :

$(1-\zeta) \sin ^{2}(t-\phi)-\zeta \sin ^{2} t=2 \sqrt{\zeta(1-\zeta)} f^{\prime}(\zeta) \sin t \sin (t-\phi)$, where

$$
\begin{aligned}
& f(\zeta)=\sqrt{\zeta(1-\zeta)-\overline{(m \zeta+n)}}, \\
& f^{\prime}(\zeta)=\frac{1-2 \zeta-m}{2 \sqrt{\zeta(1-\zeta)-(m \zeta+n)}}=\frac{1-2 \zeta-m}{2 \sqrt{\zeta(1-\zeta) \cos \phi}} .
\end{aligned}
$$

Therefore

$(1-\zeta) \sin ^{2}(t-\phi) \cos \phi-\zeta \sin ^{2} t \cos \phi=(1-2 \zeta-m) \sin t \sin (t-\phi)$.

After reduction :

$(1-\zeta) \sin (t-\phi) \cos (t-\phi)+\zeta \cos t \sin t=\frac{m \zeta(1-\zeta)}{m \zeta+n} \sin (t-\phi) \sin t \sin \phi$, $m \zeta(1-\zeta) \sin (t-\phi) \cos ^{2} t \cos (t-\phi)+n(1-\zeta) \sin (t-\phi) \cos (t-\phi)$ $+m \zeta^{2} \cos t \sin t+n \zeta \cos t \sin t+m \zeta(1-\zeta) \sin ^{2}(t-\phi) \sin t \cos t=0$. 
Dividing by $\sqrt{\zeta} \sin t$, and making use of

$$
\begin{aligned}
& x=\sqrt{\zeta} \cos t, \quad y=\sqrt{1-\zeta} \cos (t-\phi), \quad \frac{d y}{d x}=\frac{\sqrt{1-\zeta} \sin (t-\phi)}{\sqrt{\zeta} \sin t}, \\
& \text { we get } \quad x^{2} y \frac{d y}{d x}+x-x y^{2}+\frac{n}{m} y \frac{d y}{d x}+\frac{n}{m} x=0 .
\end{aligned}
$$

The integral of this differential equation runs

$$
x^{2}+y^{2}-1=\mathrm{C}\left(x^{2}+\frac{n}{m}\right), . . \cdot
$$

where $\mathrm{C}$ is a constant of integration.

The envelope is degenerated into central conic sections, having their axes along the axes of ccordinates.

\$47. In order to determine the value of (I we make use of the fact that the envelope contains the points of intersection of an ellipse corresponding to $\cos \phi=0$ with the axes of $c 0-$ ordinates ((36) of $\S 46$ is satisfied by $\cos \phi=0, \sin t=0$ or \pm 1$)$. The intersecting points of such an ellipse with the $\mathrm{Y}$-axis are given by

$$
x=0, \quad y= \pm \sqrt{1-\zeta} .
$$

$\cos \phi=0$ for the values of $\zeta$ satisfying the equation

$$
\zeta(1-\zeta)-(m \zeta+n)=0 \text {. }
$$

From these three equations and the equation of the envelope

$$
x^{2}+y^{2}-1=\mathrm{C}\left(x^{2}+\frac{n}{m}\right)
$$

we must eliminate $x, y$, and $\zeta$.

The result is :

$$
\mathrm{C}^{2}+\frac{m}{n}(1-m) \mathrm{C}+\frac{m^{2}}{n}=0 . \quad . \quad .
$$

This equation being of the second degree, we may say that the envelope is degenerated into two conic sections.

Now there are two cases :-

(1) The system of ellipses contains double curves. This will occur when $\sin \phi$ is zero for values of $\zeta$ between 0 and 1 . Therefore $m \zeta+n$ must be zero for a value of $\zeta$ between 0 and 1. In this case there are two double curves, for the system of ellipses is symmetrical in respect to the axes of coordinates. In the ends of these donble curves the enveloping conic sections intersect under right angles. When we examine the values of $\mathrm{C}$ it will be clear that one of the conic sections is an ellipse, the other an hyperbola.

Phil. Mag. S. 6. Vol. 26. No. 152. Aug.1913, Y 
(2) The system of ellipses does not contain double curves. The enveloping conic sections are ellipses, one of them being the outward envelope, the other the in ward envelope.

When the two values of $\mathrm{C}$ are equal, then the two enveloping ellipses coincide; we are in the case of the periodic motion in an ellipse.

$\S 48 . f(\zeta)$ is a linear function of $\zeta$. According to $\S 44$ this occurs when

$$
p\left(4 r+l^{2}\right)=q^{2} .
$$

In this case

$$
p \zeta^{2}+q \zeta+\frac{1}{4} l^{2}=p\left(\zeta+\frac{q}{2 p}\right)^{2}
$$

The relation between $\zeta$ and $\phi$ degenerates into

and

$$
\sqrt{\zeta(1-\zeta)} \cos \phi=\sqrt{p} \zeta+\left(\frac{q}{2 \sqrt{ } p}-\frac{1}{2} l\right)
$$

These relations are of the form

$$
\sqrt{\zeta(1-\zeta)} \cos \phi=-\sqrt{p} \zeta-\left(\frac{q}{2 \sqrt{ } p}+\frac{1}{2} l\right) \text {. }
$$

We have now

$$
\sqrt{\zeta(1-\zeta)} \cos \phi=m \zeta+n \text {. }
$$

$$
f^{\prime}(\zeta)=m \text {. }
$$

According to (34) of p. 309 for this case $\frac{d y}{d x}=$ const. Therefore: The envelope is degenerated into four straight lines, which are the sides of a restangle.

The direction of these sides may be found by means of (34). This equation may be written as follows:

It $\frac{d y}{d x}=\tan \omega$, then

$$
\frac{2 \frac{d y}{d x}}{1-\left(\frac{d y}{d x}\right)^{2}}=-\frac{1}{m}
$$

$$
\cot 2 \omega=m . \text {. . . . . . }
$$

The motion of the point takes place exactly as in the general case where no relation exists.

$\S 49 . l=0$ and $p=-q$. If we suppose $n_{y}$ to be nearly equal to $n_{x}$, then the lowest point of the surface over which the point moves is supposed to be nearly an ombilic. Now we will make, moreover, the supposition that the surface has 
two rectangular planes of symmetry; if these planes are chosen as XZ- and YZ-plane, then $l=0$. In case the planes halving the angles between the planes of symmetry just mentioned are also planes of symmetry, $p=-q$. The relation between $\zeta$ and $\phi$ takes this form :

$$
\zeta(1-\zeta) \cos ^{2} \phi=q \zeta(1-\zeta)+r .
$$

When we turn the axes of coordinates through an angle of $45^{\circ}$, then the relation between $\zeta^{\prime}$ and $\phi^{\prime}$ must bave the same form, but $q^{\prime}$ and $r^{\prime}$ will not have in general the values of $q$ and $r$.

The relation between $\zeta$ and $\phi$ may be written as follows :

$$
\zeta(1-\zeta) \sin ^{2} \phi=(1-q) \zeta(1-\zeta)-r .
$$

According to $\S 41, \zeta(1-\zeta) \sin ^{2} \phi$ is an invariant. Therefore according to $\S 46, \psi$ being $45^{\circ}$ now, we have

$$
\zeta^{\prime}\left(1-\zeta^{\prime}\right) \sin ^{2} \phi^{\prime}=(1-q)\left\{\frac{1}{4}-\zeta^{\prime}\left(1-\zeta^{\prime}\right) \cos ^{2} \phi^{\prime}\right\}-r .
$$

After reduction :

$$
\zeta^{\prime}\left(1-\zeta^{\prime}\right) \cos ^{2} \phi^{\prime}=\frac{1}{q} \zeta^{\prime}\left(1-\zeta^{\prime}\right)+\frac{q-1+4 r}{4 q} .
$$

This may be written :

$$
\zeta^{\prime}\left(1-\zeta^{\prime}\right) \cos ^{2} \phi^{\prime}=q^{\prime} \zeta^{\prime}\left(1-\zeta^{\prime}\right)+r^{\prime},
$$

where

$$
q^{\prime}=\frac{1}{q}, \quad r^{\prime}=\frac{q-1+4 r}{4 q} . \quad . \quad . \quad .
$$

$\S 50$. The case that $f(\zeta)$ is a linear function of $\zeta(\S 48)$ occurs for $l=0$ and $p=-q$ when

$$
-4 r q=q^{2}
$$

There are three possibilities, namely, $q=0, q=\infty$, and $q=-4 r$.

(1) $q=0$. The sides of the enveloping rectangles form, according to $\S 48$, where $m=0$, angles of $45^{\circ}$ with the axes of coordinates. For $r=\frac{1}{4}, \zeta=\frac{1}{2}$, $\sin \phi=0$ continually ; the rectangles have contracted into straight lines (periodic form of motion). For $r=0 \quad \cos \phi=0$ continually; the two enveloping rectangles have coincided to a square.

(2) $q=\infty$. This case may be deduced from the foregoing case by turning the axes through an angle of $45^{\circ} \quad\left(q^{\prime}=0\right)$.

(3) $q=-4 r$. We turn the axes of coordinates through such an angle that the sides of an enveloping rectangle are parallel 
to the axes of coordinates. This angle must be found from $\cot 2 \omega=2 \sqrt{r}(\S 48)$. According to $\S 46$ we have

$$
\begin{aligned}
\zeta^{\prime} & =\zeta \cos ^{2} \omega+(1-\zeta) \sin ^{2} \omega-2 \sqrt{\zeta(1-\zeta)} \cos \phi \sin \omega \cos \omega \\
& =\zeta \cos ^{2} \omega+(1-\zeta) \sin ^{2} \omega-\left(\zeta-\frac{1}{2}\right) \sin 2 \omega \cot 2 \omega \\
& =\frac{1}{2} .
\end{aligned}
$$

The envelope consists of two squares. In each of these squares we have a system of ellipses exactly as in the cases $q=0$ or $q=\infty$. These systems contain both the circle with radius $\frac{1}{2} \sqrt{2}$. Therefore we are in the case of the asymptotic form of motion; the motion approaches asymptotically to the motion in that circle. The two squares coincide for $q=-4 r=0$, and for $q=-4 r=\infty$.

\$51. The case of the degeneration into conic sections $(p=-1, \S 45)$ occurs here for $q=1$. If we examine the signification of the coefficients, it becomes clear that the surface for these values of the coefficients is a surface of revolution. The relation between $\zeta$ and $\phi$ may be written

$$
\zeta(1-\zeta) \sin ^{2} \phi=-r
$$

From the formula of $\S 41$ it follows that the axes of the ellipse are of invariable length; therefore the shape of the ellipses is invariable. In order to prove that the ellipse turns with an uniform angular velocity, and in order to calculate this angular velocity, it is necessary to caleulate $\frac{d \theta}{d t}$ from the system of equations:

$$
\begin{aligned}
\frac{d \zeta}{d t} & =m_{1} \zeta(1-\zeta) \sin \phi \cos \phi, \\
\zeta(1-\zeta) \sin ^{2} \phi & =-r, \\
\tan 2 \theta & =\frac{\zeta \sqrt{\zeta(1-\zeta)}}{1-2 \zeta} \cos \phi .
\end{aligned}
$$

It will be found that the results agree with those Prof. Korteweg arrives at.

The envelope consists of two concentric circles; this might also be deduced from the equation (37, $\S 46)$, where $C=0$.

The two enveloping circles coincide for $r=-\frac{1}{4}$; then we are in the case of the uniform motion in a parallel circle of the surface. Another special case we have when $r=0$; the motion takes place in an arbitrary meridian of the surface; one of the enveloping circles has a radius of unit length; the other has contracted into a point. 
\$52. Another interesting case occurs when $r=0$ (if we turn the axes through an angle of $45^{\circ}$, then according to $\left.\S 49, q^{\prime}=1-4 r^{\prime}\right)$.

The relation between $\zeta$ and $\phi$ becomes :

$$
\zeta(1-\zeta) \cos ^{2} \phi=q \zeta(1-\zeta)
$$

If not $\zeta=0$ or $\zeta=1$ continually, then

$$
\cos \phi= \pm \sqrt{q}
$$

All ellipses of one of these systems correspond to the same value of $\phi$. For this case $(z 0, \S 30)$ takes this form :

$$
\zeta \sin t \cos (t-\phi)+(1-\zeta) \cos t \sin (t-\phi)=0 .
$$

Therefore

$$
\zeta=\frac{\sin (\phi-t) \cos t}{\sin \phi}, \quad 1-\zeta=\frac{\cos (\phi-t) \sin t}{\sin \dot{\phi}} .
$$

$x$ and $y$ may be written as functions of $t$ :

$$
\begin{gathered}
x=\sqrt{\frac{\cos ^{3} t \sin (\phi-t)}{\sin \phi}, \quad y=\sqrt{\frac{\cos ^{3}(t-\phi) \sin t}{\sin \phi}} ;} \\
\frac{d y}{d x}=-\sqrt{\frac{\sin 2(t-\phi)}{\sin 2 t}} .
\end{gathered}
$$

The shape of the envelope as it is represented in fig. 36 (Pl. VIIl.) may be deduced from these equations. It consists of two closed parts, one having four summits, the other four cusps. For decreasing values of $q$ the two parts move towards each other; for $q=0$ they coincide into a square (fig. 33). For increasing values of $q$ one part moves to the circle with radius unity ; the other contracts into the origin ; this has taken place for $q=1$ (fig. 41).

The envelope of fig. 36 (we include the axes of coordinates) represents the limit form of the envelope of fig. 35 for a constant value of $q$, if $r$ approaches to zero. For a positive value of $r$ we have two systems of ellipses, each of them having its own envelope. For $r=0$ the two systems are no longer separated; they have in common the double curves along the axes of coordinates. We have to do with an asymptotic form of motion.

For a negative value of $r$ we have bat one system of ellipses; if this value is not too little, then the envelope consists of two concentric curves, as is represented in fig. 39 . 
We may ask in what manner the transition takes place from fig. 39 to fig. 36 . In fig. 37 the envelope is drawn for a small (negative) value of $r$; there are in the envelope 4 double points and 8 cusps. In fig. $38 r$ has a greater (negative) value; the cusps have moved towards the double points, whilst the double points move from $O$. There will belong to a given value of $q$ a certain value of $r$ for which the cusps have coincided two by two; then we are in the case of fig. 39 . In order to determine this value of $r$ for which the cusps vanish (that is to say, coincide two by two) we turn the axes of coordinates through an angle of $45^{\circ}$ and determine the intersecting points of the envelope with the $\mathrm{Y}^{\prime}$-axis. Then we get the points corresponding to $\cos \phi^{\prime}=0$ and the double points in the $Y^{\prime}$-axis. If now the cusps disappear, then for such a double point $\cos \phi^{\prime}=0 . \quad x^{\prime}=0$ for $\zeta^{\prime}=0$ and $\cos t^{\prime}=0$. By substituting $\cos t^{\prime}$ in (33) (p. 309) we get

$$
\left.\left(1-\zeta^{\prime}\right) \cos ^{2} \phi^{\prime}-\zeta^{\prime}=2 \sqrt{\zeta^{\prime}\left(1-\zeta^{\prime}\right.}\right) f^{\prime}\left(\zeta^{\prime}\right) \cos \phi^{\prime}=2 f\left(\zeta^{\prime}\right) f^{\prime}\left(\zeta^{\prime}\right) .
$$

Now we have:

$$
\begin{aligned}
f^{2}\left(\zeta^{\prime}\right) & =q^{\prime} \xi^{\prime}\left(1-\zeta^{\prime}\right)+r^{\prime} \\
2 f\left(\xi^{\prime}\right) f^{\prime}\left(\xi^{\prime}\right) & =q^{\prime}\left(1-2 \zeta^{\prime}\right)
\end{aligned}
$$

Therefore

$$
\left(1-\zeta^{\prime}\right) \cos ^{2} \phi^{\prime}-\zeta^{\prime}=q^{\prime}\left(1-2 \zeta^{\prime}\right)
$$

Eliminating $\phi^{\prime}$ from this relation, and

$$
\zeta^{\prime}\left(1-\zeta^{\prime}\right) \cos ^{2} \phi^{\prime}=q^{\prime} \zeta^{\prime}\left(1-\zeta^{\prime}\right)+r^{\prime},
$$

we get

$$
\zeta=\sqrt{\frac{r^{\prime}}{1-q^{\prime}}}
$$

If for this value of $\zeta^{\prime} \cos \phi^{\prime}=0$, then

$$
q^{\prime} \sqrt{\frac{r^{\prime}}{1-q^{\prime}}}\left(1-\sqrt{\frac{r^{\prime}}{1-q^{\prime}}}\right)+r^{\prime}=0 .
$$

Making use of $40(\$ 49)$, then we find this value of $r$ :

$$
r=(q-1)\left\{\frac{1}{(q-2)^{2}}-\frac{1}{4}\right\} \text {. }
$$

$\S 53$. Now that we have examined the special cases we shall give a review of the cases which may occur for $p=-q$ and $l=0$, for different values of $q$ and $r$. We may for that purpose follow the scheme of p. 292 . 
$q=-\infty$

$r=-\frac{1}{4} q$. The envelope of the system of ellipses is a square (fig. 23).

$-\frac{1}{4} q<r<0$. Two systems of ellipses; each of them has a rectangle as envelope (fig. 24).

$r=0$. Periodic form of motion; $\zeta=0$ or 1 continually (fig. 25).

$-\infty<q<0 . \quad r=-\frac{1}{4}(q-1)$. Periodic form of motion; $\zeta=\frac{1}{2}, \sin \phi=0$ (fig. 26).

$--\frac{1}{4} q<r<-\frac{1}{4}(q-1)$. Two systems of ellipses (fig. 27 ).

$r=-\frac{1}{4} q . \quad$ The envelope consists of two squares; asymptotic form of motion (fig. 28).

$0<r<-\frac{1}{4} q$. Two systems of ellipses (fig. 29).

$r=0$. Periodic form of motion; $\zeta=0$ or 1 continually (fig. $30)$.

$q=0$.

$r=\frac{1}{4}$. Periodic form of motion; $\zeta=\frac{1}{2}, \sin \phi=0$ (fig. 31).

$0<r<\frac{1}{4}$. Two systems of ellipses; each of them has a rectangle as envelope (fig. 32).

$r=0$. The envelope of the system of ellipses is a square (fig. 33).

$0<q<1$.

Periodic form of motion; $\zeta=\frac{1}{2}, \sin \phi=0$ (fig. 34).

$0<r<-\frac{1}{4}(q-1)$. Two systems of ellipses (fig. 35 ; in fig. $35 r$ has a very little positive value).

$r=0$. Asymptotic form of motion (fig. 36).

$-1 q<r<0$. One system of ellipses (fig. 37 belongs to a very little negative value of $r$, in fig. $38 r$ is greater, in fig. 39 still greater).

$r=-\frac{1}{4} q$. Periodic form of motion in a circle ; $\zeta=\frac{1}{2}, \cos \phi=0$ (fig. 40). 

an arbitrary straight line passing through $\mathrm{O}$ (fig. 41).

$-{ }_{4}^{1} q<r<0$. One system of ellipses; the envelope consists of two circles (fig. 42).

$r=-{ }_{4}^{1} q . \quad$ Periodic form of motion in a circle ; $\zeta=\frac{1}{2}, \cos \phi=0$ (fig. 43).

$1<q<\infty$.

$r=0$. Periodic form of motion; $\zeta=0$ or $\zeta=1$ (fig. 44).

$-\frac{1}{4}(q-1)<r<0$. Two systems of ellipses (fig. 45).

$r=-\frac{1}{4}(q-1)$. Asymptotic form of motion (fig. 46).

$-\frac{1}{4} q<r<-\frac{1}{4}(q-1)$. One system of ellipses (figs. $47,48,49$ ).

$r=-\frac{1}{4} q$. Periodic form of motion in a circle ; $\zeta=\frac{1}{2}, \cos \phi=0$ (fig. $50)$.

$q=\infty$

$r=0$. Periodic form of motion; $\zeta=0$ or $\zeta=1$ (fig. 51).

$-\frac{1}{4} q<r<0$. Two systems of ellipses; each of them has a rectangle as envelope (fig. 52).

$r=-\frac{1}{4} q$. The envelope of the system of ellipses is a square (fig. 53).

Mechanism vith three degrees of freedom.

$\S 54$. The coordinates of the representative point are given by :

$$
\begin{aligned}
& x=\frac{\sqrt{ } \alpha_{x}}{n_{x}} \cos \left(n_{x} t+2 n_{x} \beta_{x}\right), \\
& y=\frac{\sqrt{\alpha_{y \prime}}}{n_{y}} \cos \left(n_{y} t+2 n_{y} \beta_{y}\right), \\
& z=\frac{\sqrt{ } \alpha_{z}}{n_{z}} \cos \left(n_{z} t+2 n_{z} \beta_{z}\right) .
\end{aligned}
$$

These are the equations of the osculating curves. By eliminating $t$ between these equations two by two we find the projections of the osculating curves on the planes of coordinates. These projections are lissajous curves; the 
osculating curves themselves we can call Lissajous twisted curves.

Such a twisted curve remains enclosed inside a rectangular parallelopiped bounded by the planes

$$
1 x= \pm \frac{\sqrt{\alpha_{x}}}{n_{x}}, \quad y= \pm \frac{\sqrt{\alpha_{y}}}{n_{y}}, \quad z= \pm \frac{\sqrt{\alpha_{z}}}{n_{z}} .
$$

In consequence of the variability of the $\alpha$ 's this parallelopiped varies continually. According to (10) (p. 285) the following relations exist between the $\alpha$ 's :

$$
\begin{aligned}
\frac{\alpha_{x}}{p n_{x}}-\frac{\alpha_{y}}{q n_{y}} & =\text { constant } \\
\alpha_{x}+\alpha_{y} t \alpha_{z} & =\text { constant. }
\end{aligned}
$$

Therefore the vertices of the circumscribed parallelopiped move along a twisted curve, situated on an ellipsoid, whose axes lying on the axes of coordinates are in the ratio

$$
\frac{1}{n_{x}}: \frac{1}{n_{y}}: \frac{1}{n_{z}}
$$

This curve projects itself on the planes of coordinates as an ellipse or as an hyperbola; $e . g$. the $\mathrm{XY}$ projection is an ellipse if $p$ and $q$ are of different sign, an hyperbola if $p$ and $q$ are of the same sign ; it is clear that two of the projections are ellipses, the third is an hyperbola.

As the $\alpha$ 's change periodically between definite limits, the vertices will move to and fro between two extreme positions.

$\S 55$. Besides on the $\alpha$ 's the form of an osculating curve depends on the $\beta$ 's. However, for an osculating curve described in a definite parallelopiped it depends not on 3 , but only on 2 quantities, as is evident when we change the origin of time. We can get :

$$
\begin{aligned}
& x=\frac{\sqrt{\alpha_{x}}}{n_{x}} \cos \left\{n_{x} t+2 n_{x}\left(\beta_{x}-\beta_{z}\right\},\right. \\
& y=\frac{\sqrt{a_{y}} \cos }{n_{y}}\left\{n_{y} t+2 n_{y}\left(\beta_{y}-\beta_{z}\right)\right\}, \\
& z=\frac{\sqrt{\alpha_{z}}}{n_{z}} \cos n_{z} t .
\end{aligned}
$$

The form of the osculating curve evidently depends on the 
quantities $\beta_{x}-\beta_{y}$ and $\beta_{y}-\beta_{z}$. So if we put

$$
\frac{\sqrt{\alpha_{x}}}{n_{x}}=\mathrm{A}, \quad \frac{\sqrt{\alpha_{y}}}{n_{y}}=\mathrm{B}, \quad \frac{\sqrt{\alpha_{z}}}{n_{z}}=\mathrm{C}, \begin{aligned}
& 2 n_{x}\left(\beta_{x}-\beta_{z}\right)=a, \\
& 2 n_{y}\left(\beta_{y}-\beta_{z}\right)=b,
\end{aligned}
$$

then we find :

$$
\left.\begin{array}{rl}
x & =\mathrm{A} \cos \left(n_{x} t+a\right), \\
y & =\mathrm{B} \cos \left(n_{y} t+b\right), \\
z & =\mathrm{C} \cos n_{z} t
\end{array}\right\} . \cdot \cdot \cdot \cdot
$$

In the extreme parallelopipeds curves are described for which $\phi=0$ or $\pi$ (conf. $\S 24$ ). Therefore

Since

$$
2\left(p n_{x} \beta_{x}+q n_{y} \beta_{y}+r n_{z} \beta_{z}\right)=0 \text { or } \pi .
$$

$$
r n_{z}=-\left(p n_{x}+q n_{y}\right)
$$

we may write

$$
2 p n_{x}\left(\beta_{x}-\beta_{z}\right)+2 g n_{y}\left(\beta_{y}-\beta_{z}\right)=0 \text { or } \pi \text {. }
$$

Or :

$$
p a+q b=0 \text { or } \pi^{*} \text {. }
$$

$\$ 56$. Let us put in (41) $t=+\tau$ and $t=-\tau$, and let us call the values of $x, y$, and $z$ belonging to these values of $t$, respectively $x_{1}, y_{1}, z_{1}$ and $x_{2}, y_{2}, z_{2}$, then we find:

$\frac{1}{2}\left(x_{1}+x_{2}\right)=\mathrm{A} \cos a \cos n_{x} \tau, \frac{1}{2}\left(y_{1}+y_{2}\right)=\mathrm{B} \cos b \cos n_{y} \tau, z_{1}=z_{2}$.

The curve represented by (41) has therefore with respect to directions of chords parallel to the XY-plane as diameter a curve represented by the equations :

where

$$
\left.\begin{array}{l}
x_{3}=\mathrm{A}^{\prime} \cos n_{x} t \\
y_{3}=\mathrm{B}^{\prime} \cos n_{y} t \\
z=\mathrm{C} \cos n_{z} t
\end{array}\right\} . \cdot \cdot \cdot
$$

$$
\mathrm{A}^{\prime}=\mathrm{A} \cos a, \quad \mathrm{~B}^{\prime}=\mathrm{B} \cos b .
$$

To investigate the curves represented by (41) we can start from the curves represented by (42). In fig. 54 such a curve is given perspectively, in fig. 55 (continuous lines)

* The Lissajous twisted curves have been discussed by A. Righi (Il Nuovo Cimento, vols. ix. \& x., 1873). 
by projections for the simple case that we have $n_{x}: n_{y}: n_{z}=$ $1: 2: 3$ (so we are in the case $S=3$ ); the Lissajous twisted curve begins and ends in two vertices of the circumscribed parallelopiped and is described backwards and forwards.

When a curve (42) is constructed we must bear in mind that

where

$$
x=x_{3}-\delta_{1} \sin n_{x} t, \quad y=y_{3}-\delta_{2} \sin n_{y} t, \quad z=i_{3},
$$

$$
\delta_{1}=\mathrm{A} \sin a, \quad \delta_{2}=\mathrm{B} \sin b .
$$

So we can think of the curve (41) as described by a point moving along the curve (42) and vibrating at the same time according to the $X$ and $Y$ direction. From this we can see how the osculating curve changes for increasing values of $a$ and $b$. In fig. 55 the projections are represented (dotted lines) of an osculating curve for $n_{a}: n_{y}: n_{z}=1: 2: 3$ and suall values of $a$ and $b$.

Special cases.-According to $\S 24$ the periudic case occurs only for $\sin \phi=0$. The circumscribed parallelopiped does not change. The $\beta$ 's increase uniformly with the time; the osculating curve changes its form; however, invariably $\sin \phi=0$. The osculating curve is thus represented by (41); $a$ increases uniformly with the time, and $\sin (p a+q b)=0$.

For $n_{x}+n_{y}-n_{z}=\rho$ in the asymptotic case the motion approaches to $\mathrm{C}_{2}-\zeta=0$ or $\mathrm{C}_{3}-\zeta=0, \phi=\frac{\pi}{2}$ or $\frac{3 \pi}{2}(\S 23)$. For the other relations in the asymptotic case the motion approaches to $\sin \phi=0(\S 24)$.

\section{Mechanism with four degrees of freedom.}

$\S 57$. The coordinates of the representative point are given by :

$$
\left.\begin{array}{l}
x=\frac{\sqrt{\alpha_{x}}}{n_{x}} \cos \left(n_{x} t+2 n_{x} \beta_{x}\right), \\
y=\frac{\sqrt{\alpha_{y}}}{n_{y}} \cos \left(n_{y} t+2 n_{y} \beta_{y}\right), \\
z=\frac{\sqrt{\alpha_{z}}}{n_{z}} \cos \left(n_{z} t+2 n_{z} \beta_{z}\right), \\
u=\frac{\sqrt{\alpha_{u}}}{n_{u}} \cos \left(n_{u} t+2 n_{u} \beta_{u}\right) .
\end{array}\right\} . .
$$

The point moves in a space $K_{4}$. An osculating curve may be called a Lissajous wrung curve. Such a curve remains 


\section{Prof. Ratherford and Mr. Richardson : Analysis}

enclosed inside a four-dimensional parallelotope, bounded by the spaces :

$$
x= \pm \frac{\sqrt{\alpha_{x}}}{n_{x}}, \quad y= \pm \frac{\sqrt{\alpha_{y}}}{n_{y}}, \quad z= \pm \frac{\sqrt{\alpha_{z}}}{n_{z}}, \quad u= \pm \frac{\sqrt{\alpha_{u}}}{n_{z z}} .
$$

By the variability of the $\alpha$ 's the circumscribed parallelotope also changes; the vertices move backward and forward between two extreme positions along a wrung curve; this curve lies on a hyperellipsoid whose axes lying along the axes of coordinates are proportional to $\frac{1}{n_{x}}: \frac{1}{n_{y}}: \frac{1}{n_{z}}: \frac{\mathrm{J}}{n_{u}}$. The form of the Lissajous wrung curve in a deinite parallelotope depends, as is found by a change of the origin of time, on the quantities :

$$
2 n_{x}\left(\beta_{x}-\beta_{u}\right), \quad 2 n_{y}\left(\beta_{y}-\beta_{u}\right), \quad 2 n_{z}\left(\beta_{z}-\beta_{u}\right) .
$$

For the osculating curves in the extreme parallelotopes we have:

$$
2 n_{x}\left(\beta_{x}-\beta_{u}\right)+2 n_{y}\left(\beta_{y}-\beta_{u}\right) \pm 2 n_{z}\left(\beta_{z}-\beta_{u}\right)=l \pi .
$$

Furthermore, there is again a periodic case and an asymptotic case $(\S 24)$.

XIX. Analysis of the $\gamma$ Rays from Radium $D$ and Radium $E$. $B y$ Professor E. Rutherford, F.R.S., ana H. Richaldoson, M.S.**

IN a previous paper $\dagger$ we have given the results of the 1 analysis of the $\gamma$ radiation from radium $B$ and radium $C$. The radiation from the former was shown to consist of three types varying widely in penetrating power, and the latter essentially of one penutrating type. In the present paper the $\gamma$ radiation from radium $D$ and radium $E$ has been examined by a similar method. These radioactive substances are of unnsual interest and importance in considering the problem of the connexion between $\beta$ and $\gamma$ rays, since the $\gamma$ rays emitted by radium $D$ and radium $\mathbb{E}$ together are exceedingly feeble in intensity compared with the $\beta$ rays. In this respect they are in marked contrast to the products radium $B$ and radium $C$, where the $\gamma$ rays are relatively intense and comparable in energy with the $\beta$ rays.

In consequence of the short period of transformation

* Communicated by the Authors.

† Rutherford and Richardson, Phil. May. xxv. p. 722 (1913). 

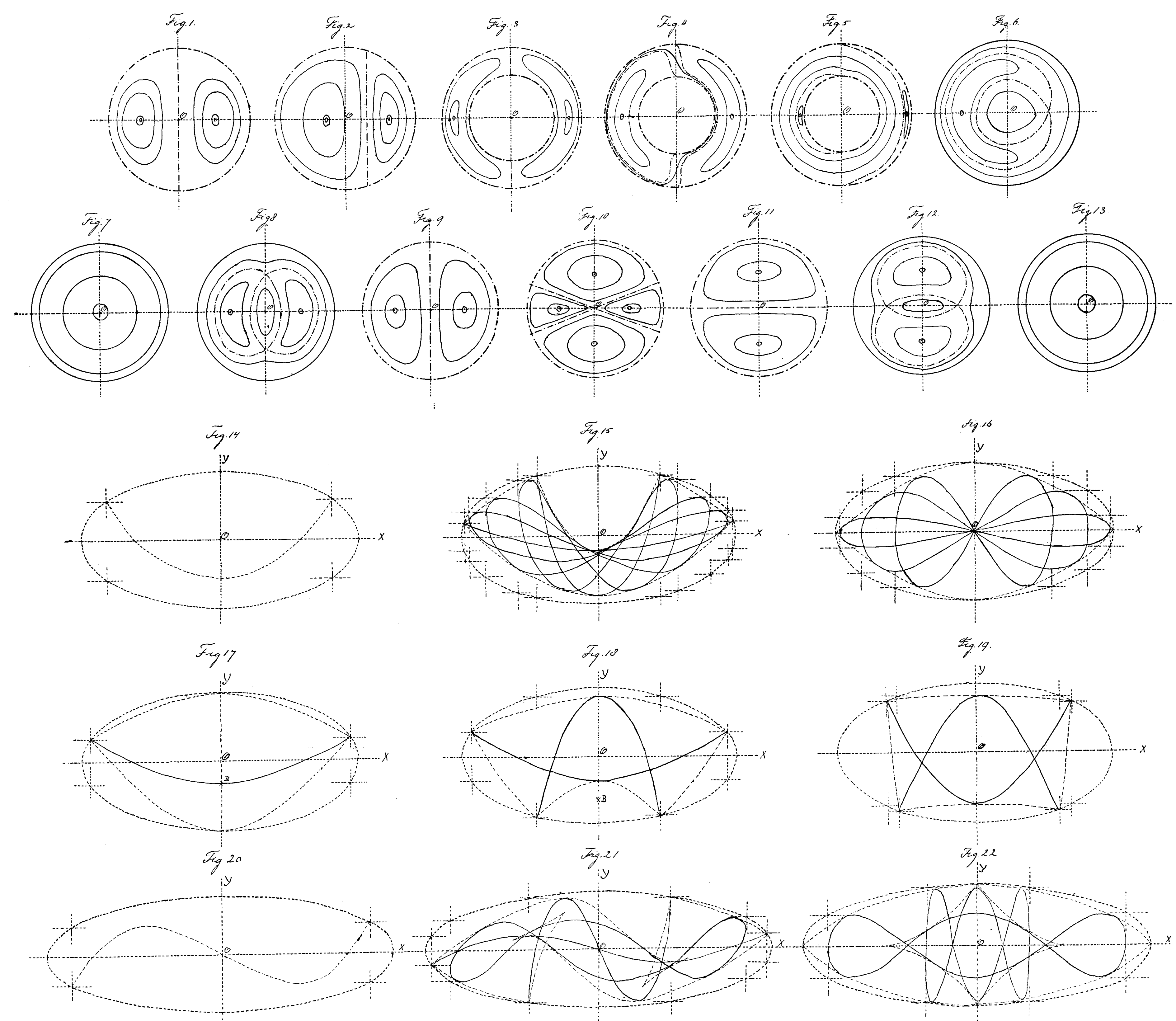


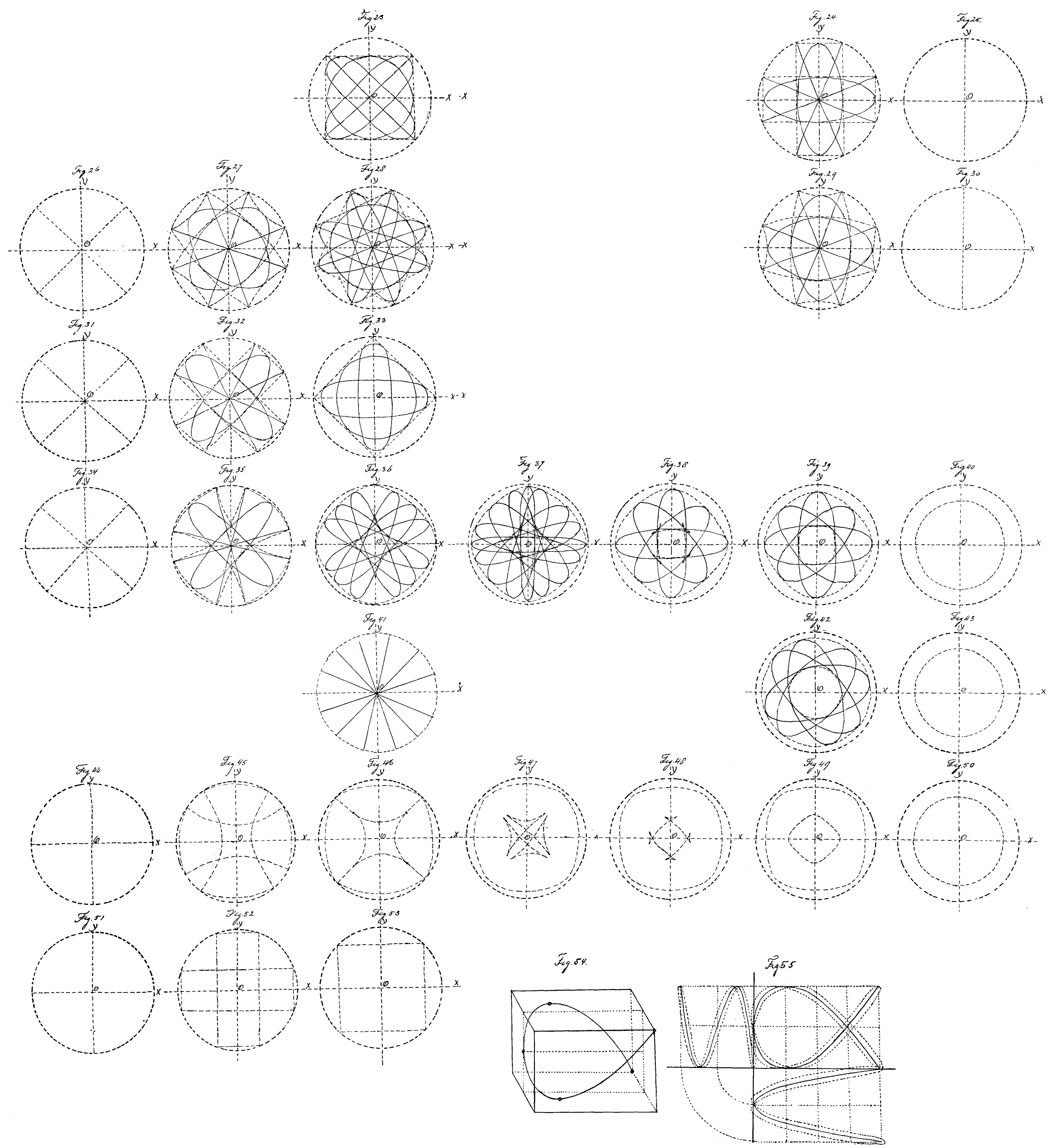

